OPEN ACCESS

Edited by:

Joshua Chou,

University of Technology Sydney,

Australia

Reviewed by:

Andrew Ekpenyong,

Creighton University, United States

Afshin Beheshti,

Space Biosciences Research of NASA Ames Research Center.

United States

*Correspondence:

Vidya Manian

vidya.manian@upr.edu

Specialty section:

This article was submitted to Cell Adhesion and Migration,

a section of the journal

Frontiers in Cell and Developmental

Biology

Received: 29 June 2021

Accepted: 12 August 2021

Published: 16 September 2021

Citation:

Manian V, Orozco-Sandoval J and Diaz-Martinez V (2021) An Integrative

Network Science and Artificial Intelligence Drug Repurposing Approach for Muscle Atrophy

in Spaceflight Microgravity.

Front. Cell Dev. Biol. 9:732370.

doi: 10.3389/fcell.2021.732370

\section{An Integrative Network Science and Artificial Intelligence Drug Repurposing Approach for Muscle Atrophy in Spaceflight Microgravity}

\author{
Vidya Manian*, Jairo Orozco-Sandoval and Victor Diaz-Martinez \\ Laboratory for Applied Remote Sensing, Imaging, and Photonics, Department of Electrical and Computer Engineering, \\ University of Puerto Rico, Mayaguez, PR, United States
}

Muscle atrophy is a side effect of several terrestrial diseases which also affects astronauts severely in space missions due to the reduced gravity in spaceflight. An integrative graph-theoretic network-based drug repurposing methodology quantifying the interplay of key gene regulations and protein-protein interactions in muscle atrophy conditions is presented. Transcriptomic datasets from mice in spaceflight from GeneLab have been extensively mined to extract the key genes that cause muscle atrophy in organ muscle tissues such as the thymus, liver, and spleen. Top muscle atrophy gene regulators are selected by Bayesian Markov blanket method and gene-disease knowledge graph is constructed using the scalable precision medicine knowledge engine. A deep graph neural network is trained for predicting links in the network. The top ranked diseases are identified and drugs are selected for repurposing using drug bank resource. A disease drug knowledge graph is constructed and the graph neural network is trained for predicting new drugs. The results are compared with machine learning methods such as random forest, and gradient boosting classifiers. Network measure based methods shows that preferential attachment has good performance for link prediction in both the gene-disease and disease-drug graphs. The receiver operating characteristic curves, and prediction accuracies for each method show that the random walk similarity measure and deep graph neural network outperforms the other methods. Several key target genes identified by the graph neural network are associated with diseases such as cancer, diabetes, and neural disorders. The novel link prediction approach applied to the disease drug knowledge graph identifies the Monoclonal Antibodies drug therapy as suitable candidate for drug repurposing for spaceflight induced microgravity. There are a total of 21 drugs identified as possible candidates for treating muscle atrophy. Graph neural network is a promising deep learning architecture for link prediction from gene-disease, and disease-drug networks.

Keywords: muscle atrophy, network measures, random walk, graph neural network, random forest, gradient boosting, preferential attachment, link prediction 


\section{INTRODUCTION}

Drug discovery is an expensive process costing an average of $\$ 1.8$ million per drug. Most drug discovery done on Earth is under a constant environment with a gravity value of $9.81 \mathrm{~m} / \mathrm{s}^{2}$. Spaceflight in satellites and the International Space Station (ISS) provides a gravitational acceleration of $1 \times 10^{-6} \mathrm{~m} / \mathrm{s}^{2}$. This is referred to as microgravity which has direct and indirect effects on an organism. The direct effects are changes in weight, distortion and deformation of organelles, and other measurable changes. The indirect changes are those that occur prior due to microgravity. Bacterial virulence and increased genetic recombination have been observed in space thereby requiring increased concentrations of antibiotics for treatment. Spaceflight environment is conducive for drug discovery, as observed in an experiment conducted on spaceflight tested a molecule Amgn0007 and sActRIIB for increasing bone mineral density in mice (Zea, 2015).

In addition to aging, muscle atrophy is slightly implicated in the etiology of chronic diseases such as diabetes, cancer, obesity, and muscular dystrophy (Kalyani et al., 2014; Muscular Dystrophy, n.d.). ${ }^{1}$ Muscle wasting also develops as a consequence of acquired immune deficiency syndrome (AIDS) (Dudgeon et al., 2006), neuromuscular disorders, and organ failure (cachexia) (Wyart et al., 2020; Rausch et al., 2021). Muscle wasting is the hallmark of cancer cachexia and is associated with serious clinical consequences such as physical impairment, poor quality of life, reduced tolerance to treatments, and shorter survival (Burckart et al., 2010). Muscle atrophy is a severe disabling clinical condition that is accompanied by cancer development in the pancreatic, lung, liver, and bladder (Bei and Xiao, 2017; Yang et al., 2018). Prolonged stay in spaceflight of up to 4 months can lead to a $17 \%$ loss of muscle mass. Muscle atrophy condition is accelerated in space due to microgravity by unloading of the muscles. Gene expression datasets have been analyzed using traditional fold change analysis and clustering methods for the identification of differentially regulated genes involved in muscle atrophy in mice flown in spaceflight (Horie et al., 2019a,b). Spaceflight simulation studies have shown differential expression of small number of microRNAs in the context of muscle physiology in response to loading (Rullman et al., 2016). Recent miRNA studies have shown that muscle degeneration with accelerated aging enhanced by exposure to space radiation and microgravity are driven by circulating miRNA and are being suggested as a potential biomarker (Malkani et al., 2020). But, advanced network analysis to identify causally related key target genes and their association with other diseases, and the application of Artificial Intelligence (AI) methods for identification of drugs suitable for treatment of muscle atrophy in spaceflight have not been performed.

Several treatments have been proposed and used for countering muscle atrophy in humans. Inhibition of a protein called myostatin has shown to result in an increase in muscle

${ }^{1}$ https://www.mayoclinic.org/diseases-conditions/muscular-dystrophy/ symptoms-causes/syc-20375388 mass (Smith et al., 2020). The drug formeterol has been used for counteracting muscle atrophy in mice in spaceflight (Ballerini et al., 2020). There are many drug candidates that can be used for treating muscle atrophy, and the use of traditional methods for drug repurposing are time consuming due to the large volume of compounds that need to be tested. AI based methods have gained importance in this pandemic era for rapid, low-cost, and effective drug repurposing (Gysi et al., 2020). AI methods rely on the fact that drugs that target one disease can target another disease with similarly functioning proteinprotein interaction networks. AI related methods are Machine Learning methods and/or Deep Learning (DL), a sub-branch of ML (Chen et al., 2020). ML methods such as Support Vector Machines (SVM), Random Forest (RF), and Gradient Boosting (Gboost) method have been used for drug repositioning to treat schizophrenia and anxiety disorders (Zhao and So, 2018). Employing ML based drug repositioning is a cost-effective way of automatizing the drug discovery process, and gaining deeper knowledge in the genetic causality of diseases, their associations, and planning preclinical trials for the selected drugs (Koromina et al., 2019; Réda et al., 2020). DL neural network architectures can explore a large amount of data, and search for similarities in several thousands of protein-protein interactions. If the input data is in the form of sequences, then Recurrent Neural Networks (RNN) are trained with the time-stamped data and used for prediction of drugs (Wang et al., 2020). Hybrid models that combine the power of Convolutional Neural Networks (CNN) and RNN have been used for drug repurposing (Xuan et al., 2019; Jarada et al., 2020). Gene protein and protein-protein interactions are generally depicted in the form of a graph, which have led to identifying disease networks and network medicine approaches for drug repurposing (Gysi et al., 2020). Network measures and evaluation metrics such as Area Under Receiver Operating Characteristic (AUROC) curves, and Area Under Precision and Recall (AUPR) have been used for network link prediction in drug discovery (Chen et al., 2018; Abbas et al., 2021). Network medicine uses graph representation for learning the patterns of protein-protein interactions. The SPOKE database (Nelson et al., 2021) is a heterogeneous knowledge graph connecting biological and clinical data from over 30 databases, that is used in this work in combination with transcriptomic datasets to create the inputs to the AI model. The Bayesian Markov blanket method applied to spaceflight transcriptomic datasets for muscle atrophy gives information on which genes are highly activated due to muscle unloading in spaceflight.

In this paper, we analyze spaceflight gene expression datasets for muscle atrophy using advanced network analysis methods and combine it with the power of AI for identifying drugs that can be repurposed for successful treatment of muscle atrophy. The rest of the paper is organized as follows. Section "Materials and Methods" presents the GeneLab datasets, and the methods used for drug repurposing, Section "Results" presents the knowledge graphs, and the link prediction results, section "Discussion" presents a discussion of the gene-disease associations, and disease-drug link predictions, and the "Conclusions" section presents the conclusions. 


\section{MATERIALS AND METHODS}

This section describes the GLDS datasets used for mining, the SPOKE database, the network analysis methods, and the ML and AI methods for link prediction. Gene expression data were downloaded from NASA GeneLab repository. The datasets were preprocessed by NASA GeneLab.

\section{GLDS-4}

Thymus lobes were extracted from young adult C57BL/6NTac mice at 8 weeks of age after exposure to spaceflight aboard the space shuttle STS-118 for a period of 13 days. Gene expression analysis demonstrate that spaceflight induces significant changes in the thymic mRNA expression of genes that regulate stress, glucocorticoid receptor metabolism, and $\mathrm{T}$ cell signaling activity (Lebsack et al., 2010). Key master regulators such as TGF- $\beta 1$ coordinating systemic response of mice to spaceflight microgravity and/or space radiation were identified in Beheshti et al. (2018).

\section{GLDS-244}

A cohort of healthy mice was implanted with subcutaneous nanofluidic delivery system ( $\mathrm{nF}$ ) of formoterol (FMT), a $\beta 2$ adrenergic receptor agonist for therapeutic treatment of skeletal muscle loss. The mice were subjected to spaceflight microgravity on ISS for 29 and 56 days before euthanizing. RNA sequencing analysis of thymus tissues showed that nF-FMT treatment mass loss in comparison to control mice (Ballerini et al., 2020).

\section{GLDS-245}

Liver tissue was extracted from the same cohort of mice used in GLDS-244 experiment. RNA sequence data was obtained from liver preserved in liquid nitrogen after dissection and stored at $-80^{\circ} \mathrm{C}$. RNA sequencing analysis of thymus tissues was done.

\section{GLDS-246}

A cohort of forty 32-weeks-old female C57BL/6NTac mice were either sham operated or implanted with vehicle or treatment-filled nDS, launched in two Transporters (20 mice per Transporter) on SpaceX-13. They were transferred to Rodent Habitats onboard the ISS, and maintained in microgravity. After 56 days, they were euthanized on the ISS and RNA samples from spleen tissue was extracted and sequencing analysis was performed.

\section{GLDS-288}

The spleens and lymph nodes were analyzed from mice flown aboard the ISS in orbit for 35 days, as part of a Japan Aerospace Exploration Agency mission. The mice were exposed to $1 \mathrm{~g}$ microgravity in the ISS. Paired end sequencing (PE36bp) was performed with NextSeq500. Whole-transcript cDNA sequencing (RNASeq) analysis of the spleen suggested that erythrocyte-related genes regulated by the transcription factor GATA1 and Tal1 were significantly down-regulated in ISS (Horie et al., 2019b).

\section{GLDS-289}

Twelve C57BL/6 J male mice (8-week-old for MHU-1 and 9-week-old for MHU-2) in transportation cage units (TCU) were launched aboard the SpaceX rocket from the KSC and transported to the ISS. After one month in spaceflight, RNA sequencing analysis showed a significantly reduced expression of cell cycle-regulating genes, resulting in reduced size of thymus. However, exposure to $1 \times \mathrm{g}$ alleviated the impairment of thymus homeostasis induced by spaceflight (Horie et al., 2019a).

\section{Gene Regulatory Network Inferencing Using Incremental Association Markov Blanket Method}

In genomics, genome to phenome analysis, and transcriptional regulatory analysis are facilitated by construction of Gene Regulatory Networks (GRNs) from gene expression datasets. The GRNs also show causal relations between the genes. Traditionally, causal relations are difficult to infer and require careful application of experimental interventions. However, causal relations can be discovered by statistical analysis of purely observational data, which is known as causal structure learning (Anand, 2009). Using Markov property, a gene is conditionally independent of all other genes except its parents, children, and children's parent variables (genes). Causal relationships are useful for combining omics data with Genome Wide Association Studies (GWAS), for inferring relationships between genotype and phenotype (Ainsworth et al., 2017).

The method used for causal relation inferencing used here is the Markov Blankets (MB) method and Bayesian Network (BN) learning (Tsamardinos et al., 2003; Ram and Chetty, 2011; Syed Sazzad et al., 2020). Joint conditional probabilities are represented by a graph in a Bayesian network, the nodes (genes) are connected by Markov property which states that a node is conditionally independent of its non-descendants, given its parents. Applying the faithfulness condition, the IAMB of any node (gene) in a $\mathrm{BN}$ is the set of parents, children, and spouses (the other parents of their common children) of the gene. In our case, each gene is a variable with a series of expression values. The Markov blanket of a gene $\mathrm{X}$ is the smallest set $\mathrm{MB}(\mathrm{X})$ containing all genes carrying information about $\mathrm{X}$ that cannot be obtained from any other gene. Association measures and conditional independent tests are applied to identify the strongly relevant genes (Pellet and Elisseeff, 2008; Bui and Jun, 2012). Hence, $\mathrm{MB}(\mathrm{T})$ is a causal structure learning algorithm useful for the discovery of regulatory interactions among genes from gene expression data. Here, MB is used to construct GRNs for regulatory relationship between genes/proteins.

\section{Gene Disease Knowledge Graph Using SPOKE}

Gene disease associations are important as the key genes of muscle atrophy are also affected by other diseases which can turn out to be lethal when transferred to the next generation. Hence, it is vital to predict which new disease can occur because of the higher activity of particular genes in the GRNs for muscle atrophy. In order to obtain the gene disease associations, we use 
the Scalable Precision Medicine Knowledge Engine (SPOKE), which is a large heterogeneous network containing multiple types of biological data capturing the essential structure of biomedicine and human health for discovery (Scalable Precision Medicine Knowledge Engine, n.d.). The maximally regulated genes identified from the GRNs are input to the SPOKE which generates all the diseases associated with these key genes obtained from the GRNs for muscle atrophy. These associations are used to construct the Gene Disease Knowledge Graph (GDKG).

\section{Network Measures}

We define a network using a graph based representation. Formally, a graph is a pair of sets $\mathrm{G}:=(\mathrm{V}, \mathrm{E})$ where $|\mathrm{V}|$ is the set of vertices (molecules, genes, proteins, nodes, points) and $|\mathrm{E}|$ is the set of edges, which is an ordered pair of $\mathrm{V}$. The graph (V, $\mathrm{E}, \mathrm{o}, \mathrm{t}$ ) is called directed, if directed edges are allowed, i.e., not all edges have reverse edges as members of $\mathrm{E}$. In a directed graph, $\mathrm{G}=(\mathrm{V}, \mathrm{E}, \mathrm{o}, \mathrm{t})$, the edges are $\mathrm{e}(\mathrm{u}, \mathrm{v}) \in \mathrm{E}$, the origin of e is denoted by $\mathrm{o}$ and the terminal $\mathrm{v}$ is denoted by $\mathrm{t}(\mathrm{v})$. In a network $\mathrm{G}=(\mathrm{V}, \mathrm{E})$, for a node $\mathrm{u}, \Gamma(\mathrm{u})=\{\mathrm{v} \mid(\mathrm{u}, \mathrm{v}) \in \mathrm{E}\}$ represents the set of neighbors of node $u$. The link prediction task in a network $G=(V, E)$ is to determine whether there is or will be a link e $(\mathrm{u}, \mathrm{v})$ between a pair of nodes $\mathrm{u}$ and $\mathrm{v}$, where $u, v \in V$, and $e(u, v) \notin E$. Similarity measures computed from neighborhoods in a graph are widely used in link prediction algorithms (Abbas et al., 2021). Random walks have been used for link prediction. Random walk methods efficiently explore neighborhoods of a node to determine a path from a starting node to a terminal node. Probabilities are usually used to select the next neighboring node in the path. Biomolecular networks are complex and random walks are an efficient way for exploring them (Costa and Travieso, 2007; Janwa et al., 2019). A semi-supervised scalable feature learning method is proposed in Grover and Leskovec (2016), where the authors develop a family of biased random walks resulting in a flexible search space of nodes for link prediction. We have used this method to obtain the highest ranked nodes for possible links between muscle atrophy genes and their associated diseases. Apart from random walk, we have computed the preferential attachment network measure to obtain possible gene-disease and disease-drug associations. Preferential Attachment is the multiplication of the degrees of nodes $\mathrm{u}$ and $\mathrm{v}: P A(u, v)=$ $|\Gamma(u)||\Gamma(v)|$

\section{Graph Neural Network for Prediction of Gene-Disease Associations}

A deep Graph Neural Network (GNN) architecture consisting of multiple layers and hundreds of nodes is constructed and takes as input the GDKG constructed as described in section "Gene Disease Knowledge Graph Using SPOKE." This graph $G=(V, E)$ is multimodal and heterogeneous with $\mathrm{N}$ nodes vi $\epsilon \mathrm{V}$ is the set of nodes representing proteins or genes, and diseases. The edges $\mathrm{E}$ represents gene-disease associations. The link prediction task is to predict whether there will, is, or will be a link e $(\mathrm{u}, \mathrm{v})$ between a pair of nodes $\mathrm{u}$ and $\mathrm{v}$, where $\mathrm{u}, \mathrm{v} \in \mathrm{V}$ and $e(u, v) \notin E$. A link prediction problem is setup on the GDKG representation for identifying links between genes and the diseases associated with it. The GNN is a three layer model. The edge features of the GDKG are the input to the input layer of the GNN. The hidden layer consists of 300 neurons with "tanh" activation function. Limitedmemory Broyden-Fletcher-Goldfarb-Shanno (lbfgs) solver from the sktlearn library is used for link prediction. It approximates the second derivative matrix updates with gradient evaluations. It stores only the last few updates, so it saves memory. The output of the GNN is a matrix consisting of new predicted edges.

\section{Random Forest Method}

The RF is a classifier using the ensemble learning algorithm on a multitude of decisions trees constructed at training time. It trains decision trees using random sampling with replacement. For each node in the base decision tree, random forest randomly chooses an attribute subset including $\mathrm{k}(\mathrm{k} \leq \mathrm{m})$ attributes from the attribute set of the node (including $\mathrm{m}$ attributes), and then, chooses the best attribute from the subset to split samples (the optimal judgment is usually based on the minimum of a Gini index). The split process will be repeated until the split termination condition is satisfied (generally, the Gini index is small enough), and the model integrated by multiple decision trees is a random forest ( $\mathrm{Wu}$ et al., 2018). Each tree emits a prediction, and the class with the most votes becomes the model's prediction. It is based on the principle that many uncorrelated models (trees) operating as a committee will outperform any of the individual constituent models.

\section{Gradient Boosting Classifier}

The Gboost classifier is also an ensemble learning method similar to random forest except that it trains one tree at a time. This additive model (ensemble) works in a forward stage-wise manner, introducing a weak learner to improve the shortcomings of existing weak learners (Li et al., 2016). In Gboost, shortcomings are identified by gradients. Whereas in Adaboost, shortcomings are identified by high-weight data points. Both high-weight data points and gradients tell us how to improve our model. RF combine results at the end of the process (by averaging or "majority rules") while Gboost combines results along the way. Gboost is not a best method if there is lot of noise in the data, as it results in overfitting. The parameters are harder to tune than RF.

\section{Disease Drug Link Prediction}

The top ranked disease associations from the GDKG that have highest probability of predicted and existing links are selected. For each of these disease up to ten drugs are chosen from the DrugBank database (Drugbank online, n.d.). ${ }^{2}$ There are multiple drugs that are used for several diseases. An adjacency matrix with rows for diseases and columns for drugs is constructed. The Disease Drug Knowledge Graph (DDKG) is generated and the link prediction algorithm is run on this graph. This results in top ranked drugs with highest probability that can be repurposed for muscle atrophy. The choice of the best drug also depends on the diagnostics and prognostics of the disease, hence the most prevalent comorbidities with muscle atrophy is considered for the drug selection. Drug selection is also a very sensitive process and requires clinical intervention, hence, we provide

${ }^{2}$ https://go.drugbank.com/ 
a list of drugs that may be considered for treatment of this condition in spaceflight.

Figure 1 shows the sequence of steps followed for constructing GDKG, DDKG, and link prediction for drug repurposing in muscle atrophy. The predicted drugs have the highest probability for muscle atrophy treatment in spaceflight. Once the DDKG is constructed any machine learning approach for link prediction can be used for predicting probable drugs. The network feature extraction method used here is based on random walks, which can be replaced with other local or global graph similarity based indices such as common neighbors, Jaccard index, Sorensen index, preferential attachment, Adamic-Adar index, resource allocation index, hub promoted index, Leicht-Holme-Newman index, parameter dependent index, local affinity structure index, individual attraction index, mutual information index, functional similarity weight, and local neighbors link index, Katz index, and page rank (Fire et al., 2011; Mutlu et al., 2020). In this case, we found random walk and preferential attachment to give better results than the other features.

\section{Metrics for Evaluation of Link Prediction Methods}

There are several measures to evaluate the performance of link prediction methods. The Receiver Operating Curve (ROC) represents the performance trade-off between true positive and false positives at different decision boundary thresholds. AUROC is the Area Under the Receiver Operating Characteristics (AUROC) value, which is the area under the plot between True Positive Rate (TPR) and the False Positive Rate (FPR). It represents the trade-of between $\mathrm{TP}$ and $\mathrm{FP}$ prediction rates. The TPR is also known as sensitivity, recall, or probability of

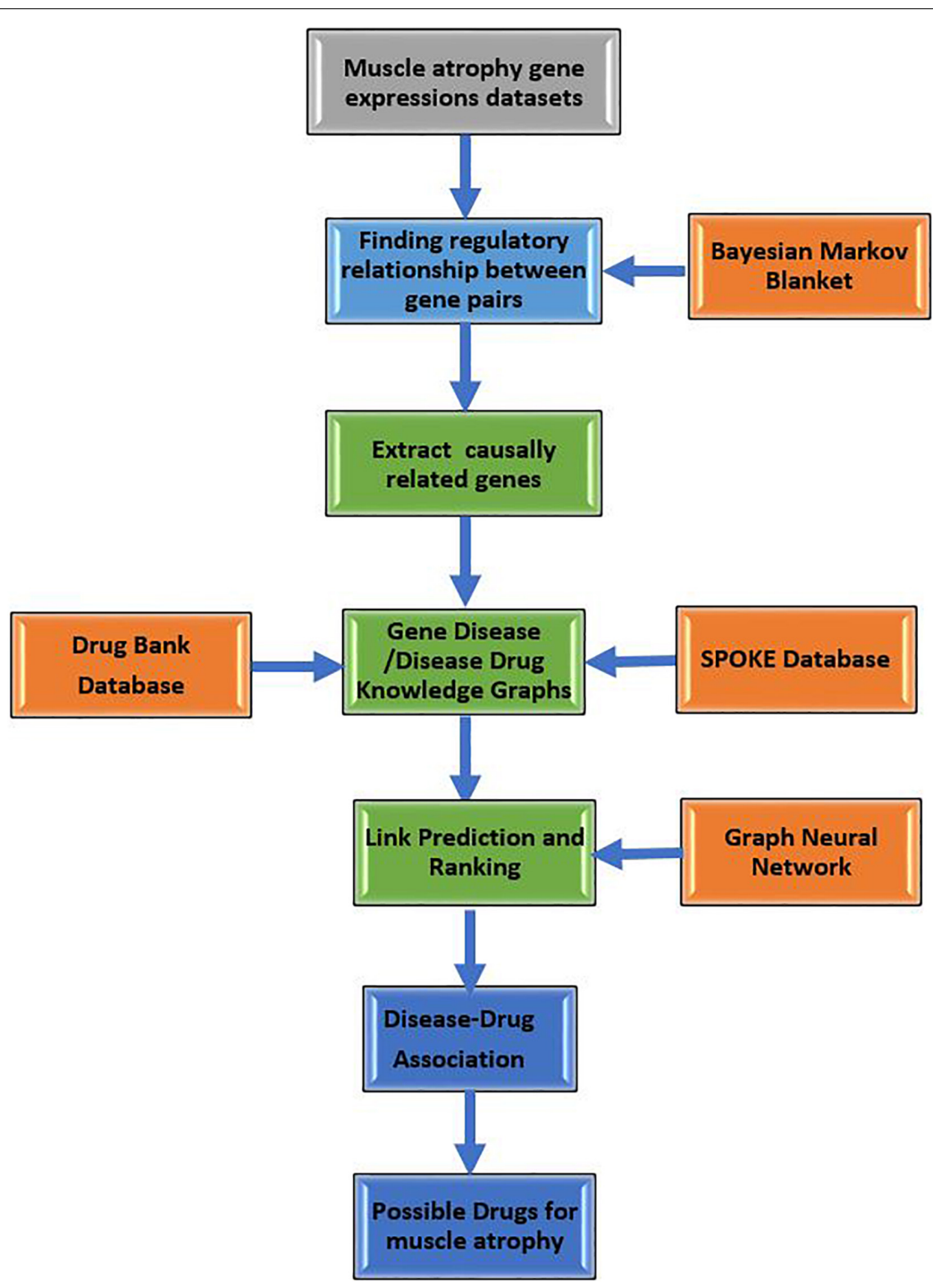

FIGURE 1 | Flow diagram showing sequence of steps followed for constructing GDKG, DDKG, and link prediction for drug repurposing to treat muscle atrophy in spaceflight microgravity. 
detection. AUROC measures the separability of the classifier and is therefore a vital metric (Yang et al., 2015).

\section{Computational Network Measures}

The network measures used to analyze the GDKG and DDKG networks include spectral gap, girth or diameter, and density. Measures computed on the gene nodes and drug nodes are degree distribution, neighborhood connectivity, and subgraph centrality (Biggs, 1993).

Spectral gap: For a graph $G$, the Laplacian eigenvalues can be ordered as $1=\left|\lambda_{1}\right| \geq\left|\lambda_{2}\right| \geq \cdots \geq|\lambda \mathrm{n}|$ (G may be directed or undirected, weighted or unweighted, simple or not). The Spectral gap is defined as: $\delta_{\lambda}=\left|\lambda_{1}\right|-\left|\lambda_{2}\right|$. By normalizing the Laplacian matrix of G, the eigenvalues are $\lambda_{1} \geq \lambda_{2} \geq \cdots \geq \lambda \mathrm{n}>0$, and the Laplacian spectral gap will be: $\delta_{\lambda}=1-\left|\lambda_{2}\right|$. The spectral gap is also known as a random walk, in terms of this concept $\lambda_{2}$ is the most important eigenvalue. Note that if the spectral gap is 0 , which means $\lambda_{2}=1[\Gamma$ is not (strongly) connected or if $\Gamma$ is bipartite], this means a typical random walk will not converge to a unique distribution or dominant eigenvector. As long as the spectral gap is greater than 0 , which means $\left|\lambda_{2}\right|<1$, then the random walk converges to a unique dominant eigenvector, and the spectral gap measures the rate of convergence, the larger the spectral gap (the smaller $\left|\lambda_{2}\right|$ ), the better the network flow [large $h(G)$, diffusion, mixing, random walk, expansion, sparsity, and other highly desirable properties of the network G].

Girth of a graph is the smallest positive integer $r$ such that $\operatorname{Trace}\left(\mathrm{A}^{\mathrm{r}}\right)>0$. Let $\mathrm{d}=\mathrm{d}(\mathrm{G})$ be the smallest integer (if it exists) so that for every pair of vertices $(\mathrm{u}, \mathrm{v})$ there is a walk of length at most $\mathrm{d}$ from $\mathrm{u}$ to $\mathrm{v}$. Then $\mathrm{d}(\mathrm{G})$ is called the diameter or maximum eccentricity of the graph G.
Density of a graph is the ratio between the number of edges and the number of possible edges. Density is a measure of the compactness of a module (subnetwork) and measures the connectivity strength of pairs of genes in the module (Hussain Ahmed et al., 2020).

The clustering coefficient models the degree of clustering of a subset of nodes. A node is selected, and we see how connected the node is with other nodes that are also connected to it. The clustering coefficient is used to characterize network modularity, which is a strength of measure of a network division into modules or groups.

Degree distribution is the number of neighbors connected to a node; in other words, it is the number of edges incident on a node. The degree distribution can give information about the structure of a network. The networks can be directed or undirected. In the undirected case, the degree of node $i$ is the number of connections it has, and it can be represented as an adjacency matrix, with the sum over all nodes. For directed graphs, there are two types of degree distributions: in-degree, which is the number of connections entering the node, and outdegree, which is the number of outgoing connections. In this case, the degree distribution is computed for the genes in the GDKG and for the drugs in the DDKG.

Subgraph centrality of a node is a weighted sum of closed walks of different lengths in the network starting and ending at a node. Centrality measures are used widely in biological networks to infer protein-protein interactions and identify essential proteins (Opsahl et al., 2010).

\section{Implementation}

The GRN inferencing method using MB is implemented in R. This method is used to construct the GRN's for each of the

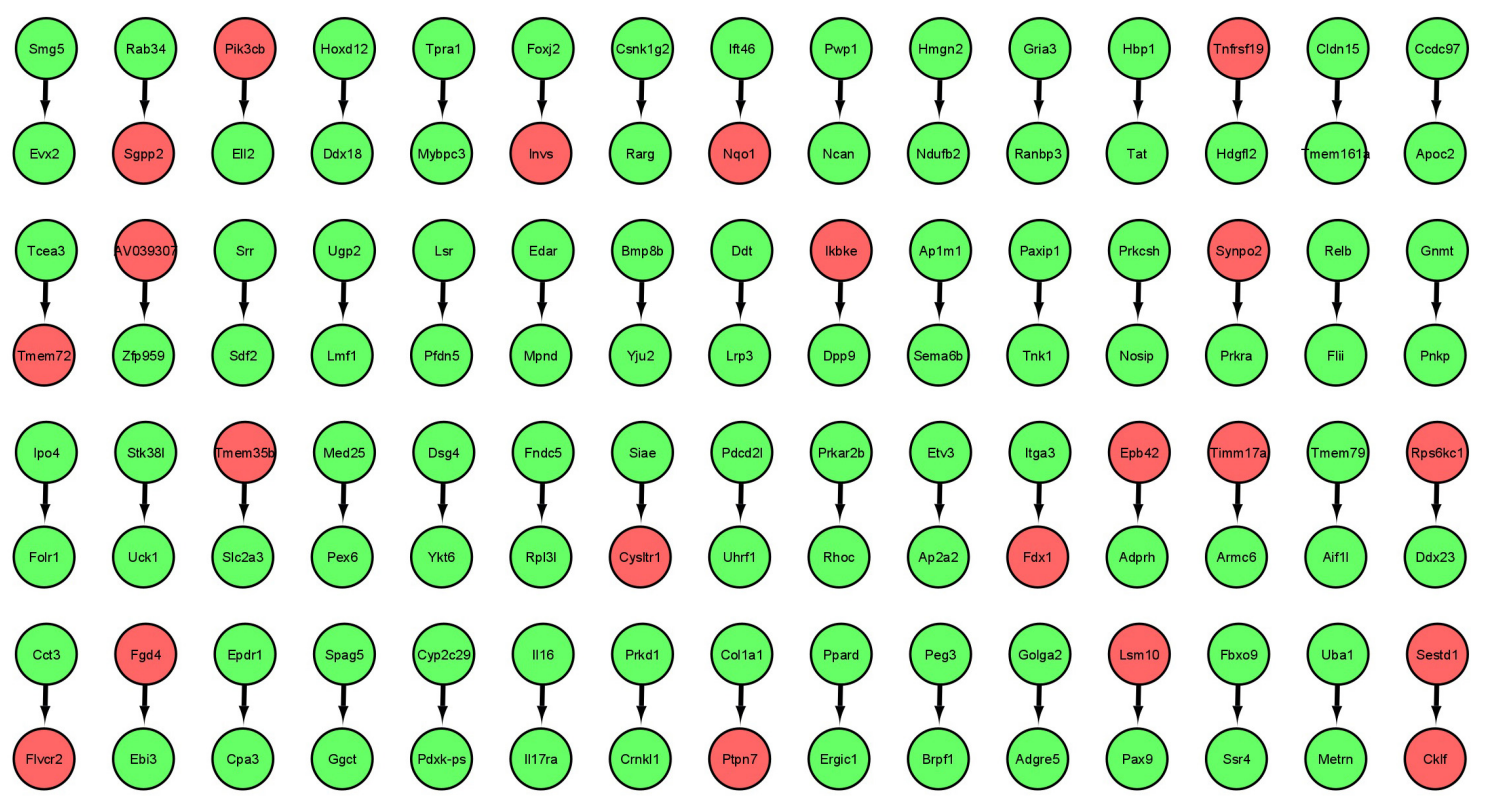

FIGURE 2 | Markov Blanket Gene Regulatory Network for GLDS-246 dataset. Red colored circles are genes with higher regulatory activity selected for constructing the GDKG. 
TABLE 1 | Maximally regulated genes for muscle atrophy from the spaceflight Genelab datasets GLDS-4, GLDS-244, GLDS-245, GLDS-246, GLDS-288, and GLDS-289 selected by Markov Blanket network analysis.

\begin{tabular}{|c|c|c|c|}
\hline Gene name & $\begin{array}{c}\text { Degree } \\
\text { distribution }\end{array}$ & $\begin{array}{l}\text { Neighborhood } \\
\text { connectivity }\end{array}$ & $\begin{array}{l}\text { Subgraph } \\
\text { centrality }\end{array}$ \\
\hline AAMP & 16 & 19.6875 & $1,066,536$ \\
\hline ABCA2 & 7 & 63.28571 & $2,032,188$ \\
\hline ABCA6 & 12 & 40.08333 & $2,517,512$ \\
\hline ACTA2 & 13 & 33 & $1,265,017$ \\
\hline ADAMTS8 & 2 & 129 & $483,438.2$ \\
\hline AFP & 6 & 59.5 & $1,090,336$ \\
\hline AGA & 2 & 129 & $483,438.2$ \\
\hline AGBL5 & 7 & 63.28571 & $2,032,188$ \\
\hline ALDH1L2 & 2 & 129 & $483,438.2$ \\
\hline ANP32A & 8 & 44.375 & $1,095,830$ \\
\hline APIP & 6 & 15 & $51,842.77$ \\
\hline ARFIP1 & 2 & 129 & $483,438.2$ \\
\hline ARHGEF7 & 7 & 63.28571 & $2,032,188$ \\
\hline ARPC2 & 16 & 19.6875 & $1,066,536$ \\
\hline ASB6 & 2 & 129 & $483,438.2$ \\
\hline ASPH & 2 & 129 & $483,438.2$ \\
\hline ATF3 & 17 & 10 & $204,496.1$ \\
\hline ATF7IP & 23 & 27.6 & $3,275,739$ \\
\hline BAG3 & 11 & 31.36364 & $839,991.3$ \\
\hline BAIAP2 & 5 & 55 & $543,055.9$ \\
\hline BATF3 & 17 & 10 & $204,496.1$ \\
\hline BCL6 & 8 & 56.125 & $2,101,026$ \\
\hline BRD4 & 7 & 63.28571 & $2,032,188$ \\
\hline $\mathrm{CCNI}$ & 2 & 129 & $483,438.2$ \\
\hline ССT5 & 2 & 129 & $483,438.2$ \\
\hline CD33 & 6 & 52.66667 & $697,061.2$ \\
\hline CDKN1B & 13 & 33.84615 & $1,540,483$ \\
\hline CEBPB & 3 & 14.66667 & $8,307.823$ \\
\hline CHFR & 8 & 51.57143 & $1,029,718$ \\
\hline COL2OA1 & 2 & 129 & $483,438.2$ \\
\hline CRTC2 & 8 & 59.5 & 1090336 \\
\hline CRYL1 & 2 & 129 & $483,438.2$ \\
\hline CYSLTR1 & 2 & 129 & $483,438.2$ \\
\hline DDB1 & 2 & 129 & $483,438.2$ \\
\hline DHX8 & 7 & 63.28571 & $2,032,188$ \\
\hline DIS3 & 7 & 63.28571 & $2,032,188$ \\
\hline DNMT3B & 16 & 40.61538 & $2,985,855$ \\
\hline DTX3 & 5 & 11.4 & $18,321.32$ \\
\hline DUSP6 & 2 & 129 & $483,438.2$ \\
\hline ECD & 2 & 129 & $483,438.2$ \\
\hline EEF1B2 & 8 & 47 & $1,208,616$ \\
\hline ELP5 & 2 & 129 & $483,438.2$ \\
\hline EPB42 & 2 & 129 & $483,438.2$ \\
\hline ERP27 & 7 & 44.85714 & $686,034.2$ \\
\hline FADS1 & 46 & 16.34091 & $4,995,617$ \\
\hline FAM167A & 13 & 6.615385 & $49,473.38$ \\
\hline FAM20B & 2 & 129 & $483,438.2$ \\
\hline FCER1G & 11 & 12.81818 & $141,251.6$ \\
\hline FGD4 & 5 & 58 & $592,697.7$ \\
\hline FGG & 3 & 92.33333 & $551,896.8$ \\
\hline FLVCR2 & 2 & 129 & $483,438.2$ \\
\hline
\end{tabular}

(Continued)
TABLE 1 | (Continued)

\begin{tabular}{|c|c|c|c|}
\hline Gene name & $\begin{array}{c}\text { Degree } \\
\text { distribution }\end{array}$ & $\begin{array}{l}\text { Neighborhood } \\
\text { connectivity }\end{array}$ & $\begin{array}{l}\text { Subgraph } \\
\text { centrality }\end{array}$ \\
\hline GBF1 & 22 & 27 & $3,570,302$ \\
\hline GLI3 & 13 & 38.07692 & $2,695,724$ \\
\hline GNA13 & 4 & 81.25 & 875,946 \\
\hline GULP1 & 2 & 129 & $483,438.2$ \\
\hline HAUS4 & 2 & 129 & $483,438.2$ \\
\hline HEMGN & 7 & 65.6 & $906,075.6$ \\
\hline $\mathrm{HIC1}$ & 8 & 11.25 & $43,296.84$ \\
\hline IKBIP & 2 & 129 & $483,438.2$ \\
\hline IKBKE & 11 & 13 & $134,489.2$ \\
\hline IMP3 & 3 & 14.66667 & $8,307.823$ \\
\hline ING5 & 11 & 12.72727 & $194,840.4$ \\
\hline INPP4A & 2 & 129 & $483,438.2$ \\
\hline INVS & 7 & 63.28571 & $2,032,188$ \\
\hline IQSEC1 & 5 & 58.4 & $625,550.6$ \\
\hline LIMCH1 & 12 & 41.08333 & $2,669,384$ \\
\hline LLGL2 & 2 & 129 & $483,438.2$ \\
\hline LMAN2 & 24 & 22.45833 & $2,625,946$ \\
\hline LOXL3 & 2 & 129 & $483,438.2$ \\
\hline LUZP1 & 7 & 63.28571 & $2,032,188$ \\
\hline MAP3K8 & 6 & 52.5 & 811,120 \\
\hline MBNL1 & 11 & 44.55556 & $1,288,207$ \\
\hline MGAT5 & 12 & 34.45455 & $1,180,487$ \\
\hline MMACHC & 7 & 44 & $701,632.3$ \\
\hline MTHFD1L & 13 & 40.76923 & $2,703,941$ \\
\hline MTSS1 & 5 & 57.8 & $587,272.6$ \\
\hline MTUS1 & 2 & 129 & $483,438.2$ \\
\hline MVP & 5 & 58.2 & $651,412.9$ \\
\hline NAGLU & 5 & 60.4 & $617,943.7$ \\
\hline $\mathrm{NOTCH} 1$ & 34 & 21.58065 & $5,186,101$ \\
\hline NPM1 & 10 & 10.6 & $76,055.24$ \\
\hline NQO1 & 9 & 11.22222 & $50,685.27$ \\
\hline NSUN6 & 15 & 24.4 & $920,237.7$ \\
\hline NUCKS1 & 13 & 34.30769 & $1,624,638$ \\
\hline NUFIP2 & 5 & 55 & $543,055.9$ \\
\hline NUP35 & 2 & 129 & $483,438.2$ \\
\hline ODF2L & 2 & 129 & $483,438.2$ \\
\hline PCBD2 & 15 & 21.4 & $1,100,177$ \\
\hline PCSK5 & 3 & 87.66667 & $510,939.4$ \\
\hline PDCD11 & 10 & 47.7 & $2,313,937$ \\
\hline PDLIM5 & 8 & 59.5 & $1,090,336$ \\
\hline PDPR & 2 & 129 & $483,438.2$ \\
\hline PIK3C2A & 9 & 52 & $2,218,15$ \\
\hline PIКЗСВ & 2 & 129 & $483,438.2$ \\
\hline PLA2G7 & 4 & 71.75 & $599,936.5$ \\
\hline PLG & 18 & 7.5 & $97,555.15$ \\
\hline PLXNA4 & 2 & 129 & $483,438.2$ \\
\hline POLB & 2 & 129 & $483,438.2$ \\
\hline PPAN & 3 & 86.33333 & $485,964.5$ \\
\hline PPFIA1 & 7 & 63.28571 & $2,032,188$ \\
\hline PPP1CB & 7 & 56.16667 & $954,019.9$ \\
\hline PTCD3 & 2 & 129 & $483,438.2$ \\
\hline PTEN & 36 & 18.77778 & $5,440,125$ \\
\hline
\end{tabular}


TABLE 1 | (Continued)

\begin{tabular}{|c|c|c|c|}
\hline Gene name & $\begin{array}{c}\text { Degree } \\
\text { distribution }\end{array}$ & $\begin{array}{l}\text { Neighborhood } \\
\text { connectivity }\end{array}$ & $\begin{array}{l}\text { Subgraph } \\
\text { centrality }\end{array}$ \\
\hline PTPN22 & 36 & 16.76471 & $2,848,757$ \\
\hline PTPN7 & 5 & 42.2 & $404,444.5$ \\
\hline PVR & 12 & 11.91667 & $142,451.7$ \\
\hline RAB1B & 6 & 10.66667 & $25,252.28$ \\
\hline RAN & 13 & 28.30769 & $1,013,655$ \\
\hline RNF13 & 4 & 9 & $5,753.418$ \\
\hline RPS24 & 2 & 129 & $483,438.2$ \\
\hline RPS6KC1 & 2 & 129 & $483,438.2$ \\
\hline RSRP1 & 2 & 6 & $1,857.078$ \\
\hline RUSC2 & 7 & 63.28571 & $2,032,188$ \\
\hline SAMD10 & 3 & 14.66667 & $8,307.823$ \\
\hline SCAF8 & 11 & 45.72727 & $2,543,016$ \\
\hline SEPHS1 & 2 & 129 & $483,438.2$ \\
\hline SESTD1 & 9 & 50.55556 & $2,117,183$ \\
\hline SFXN1 & 2 & 129 & $483,438.2$ \\
\hline SGPP2 & 2 & 129 & $483,438.2$ \\
\hline SH3TC2 & 2 & 129 & $483,438.2$ \\
\hline SIN3B & 7 & 63.28571 & $2,032,188$ \\
\hline SLC12A6 & 8 & 56.625 & $2,121,072$ \\
\hline SLC37A1 & 18 & 23.83333 & $1,425,904$ \\
\hline SLC39A1 & 6 & 59.5 & $1,090,336$ \\
\hline SLCO2A1 & 13 & 33.41667 & $1,510,348$ \\
\hline SNX25 & 2 & 129 & $483,438.2$ \\
\hline SPATA6 & 2 & 129 & $483,438.2$ \\
\hline SPG11 & 7 & 63.28571 & $2,032,188$ \\
\hline SPRY4 & 22 & 24.42857 & $2,141,129$ \\
\hline ST14 & 2 & 129 & $483,438.2$ \\
\hline SUB1 & 7 & 34.42857 & $498,913.4$ \\
\hline SYNPO2 & 12 & 40.08333 & $2,517,512$ \\
\hline TAF15 & 1 & 10 & 953.6951 \\
\hline TCF25 & 24 & 19.5 & $1,637,001$ \\
\hline THUMPD3 & 2 & 129 & $483,438.2$ \\
\hline TMEM106B & 7 & 8 & $16,732.19$ \\
\hline TMEM123 & 6 & 59.5 & $1,090,336$ \\
\hline TMTC1 & 8 & 56.625 & $2,121,072$ \\
\hline TNFRSF19 & 12 & 38.7 & $1,291,127$ \\
\hline TPK1 & 6 & 67 & $945,841.4$ \\
\hline TPM4 & 2 & 129 & $483,438.2$ \\
\hline TPMT & 10 & 28.8 & $663,140.7$ \\
\hline TRIM25 & 4 & 14.25 & $42,289.27$ \\
\hline TTPAL & 2 & 129 & $483,438.2$ \\
\hline TXNL1 & 5 & 60.4 & $617,943.7$ \\
\hline UBA3 & 2 & 129 & $483,438.2$ \\
\hline UBASH3A & 23 & 23.7619 & $2,102,919$ \\
\hline USP37 & 2 & 129 & $483,438.2$ \\
\hline VPS37A & 4 & 12.75 & $16,391.32$ \\
\hline XRCC1 & 2 & 129 & $483,438.2$ \\
\hline ZBTB37 & 2 & 129 & $483,438.2$ \\
\hline ZFPM2 & 17 & 32.47059 & $3,141,241$ \\
\hline ZMIZ1 & 40 & 17.97368 & $4,230,617$ \\
\hline ZMYND11 & 2 & 129 & $483,438.2$ \\
\hline
\end{tabular}

The network measurements computed for these genes from the GDKG is given in columns 2-4. spaceflight muscle atrophy datasets. The GDKG construction is done using SPOKE database and its adjacency matrix is created in MS Excel. The drug disease adjacency matrix is first constructed in MS Excel after downloading the drugs for each disease from drug bank. Cytoscape is used to visualize the networks. Exhaustive search method from the GridSearchCV library is used to estimate the best parameters for the link prediction methods. For the gene disease link prediction, the parameters chosen for the RF method are: depth of 15 for the RF with 500 estimators, and a learning rate of 0.2 for Gboost method. The GNN is a deep neural network with 10 layers consisting of 100 hidden nodes in each layer, it uses "relu" for activation, and Adam solver. For disease drug link prediction, the estimated parameters are a depth of 5 for the RF method with 500 estimators, and a learning rate of 0.2 for the Gboost method. The GNN has 10 layers with 100 hidden nodes in each layer, uses "relu" for activation, and Lbfgs solver. The GridSearchCV library also estimates the best number of split for cross validation, as well. In our implementation, we have chosen 10-fold cross validation. The computation of network features, and graph features are implemented in Python using the libraries networkX, node2vec, pandas, numpy, and sklearn. The implementations are available in github. ${ }^{3}$

\section{RESULTS}

Results of GRN inferencing, knowledge graph construction, and the training and validation of link prediction methods are presented below.

\section{GRN Inferencing and Construction of Knowledge Graphs}

The gene expression values corresponding to spaceflight experiments are extracted from the excel files for the six GeneLab datasets and input to the MB GRN inferencing method. The number of values range from three to eight. Figure 2 shows the MB GRN for GLDS-246 dataset. Table 1 gives the list of the common genes identified from the GRNs that are highly activated due to muscle atrophy in spaceflight microgravity from the GLDS-4, 244, 245, 246, 288, and 289 datasets. Red nodes are genes with higher regulatory activity for muscle atrophy selected for constructing the GDKG. Figure 3 shows the GDKG constructed from the highly activated genes from the six datasets and the SPOKE database. Figure 4 shows the complete DDKG. Table 2 lists the diseases identified from the GDKG. The GDKG in matrix notation is of dimension $299 \times 1195$, where 299 is the number of nodes and 1,195 is the number of edges.

\section{Training and Validation of Link Prediction Methods}

The Preferential Attachment (PA) method outputs the predicted links from the GDKG and DDKG matrices. These matrices are divided into training and validation sets. The training network is

\footnotetext{
${ }^{3}$ https://github.com/jjorozco777/An-integrative-Network-Science-andArtificial-Intelligence-Drug-Reporposing-Approach
} 


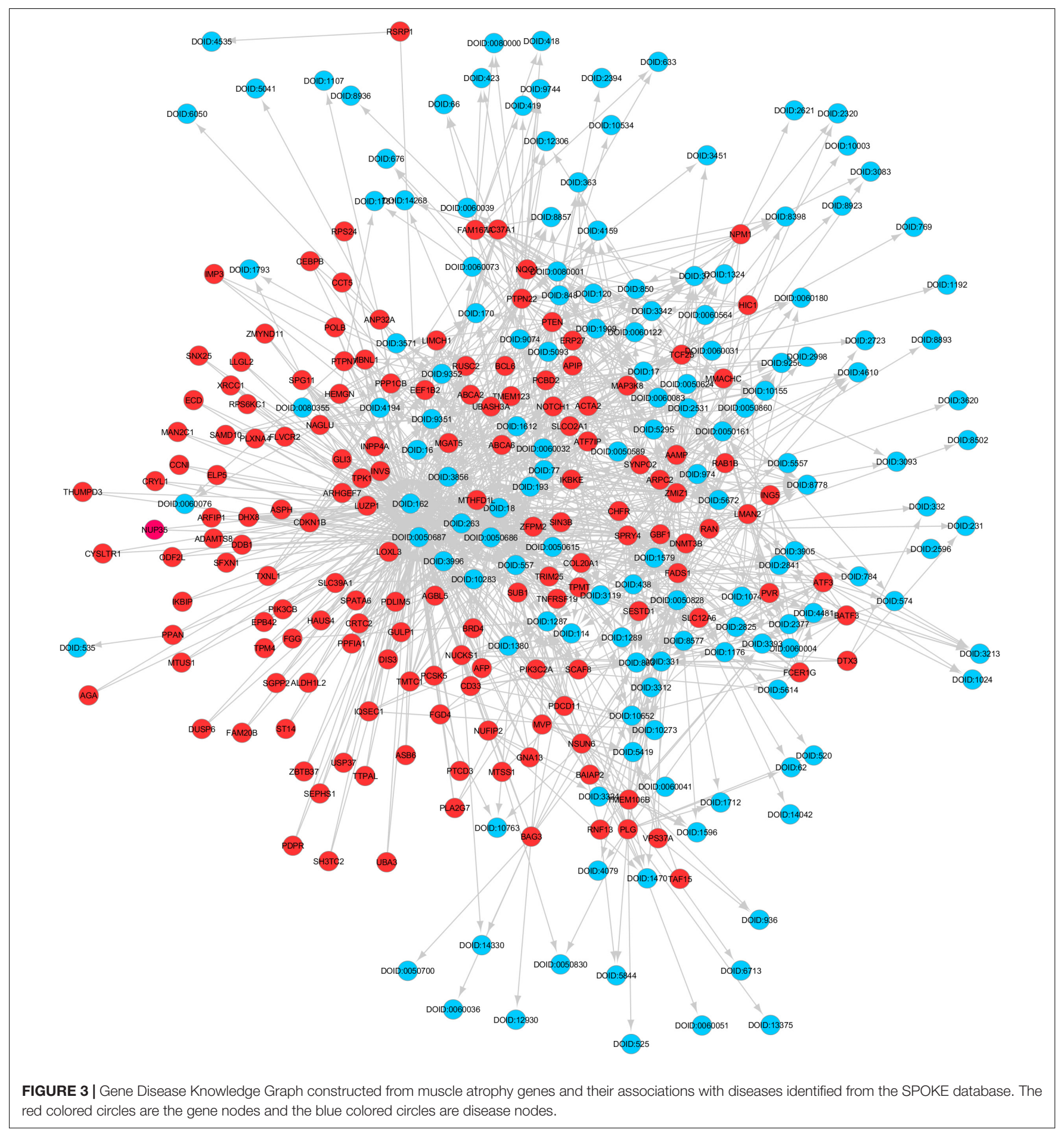

of size $299 \times 298$ which is input to the random walk network feature extraction method. The input matrix to the three link prediction methods of RF, Gboost, and GNN is a network measure matrix of dimension $2199 \times 100$, where 2,199 is the number of pairs of nodes, and 100 is the number of random walk features. Overall, the three link prediction methods perform better than PA method. The output of all the link prediction methods is a matrix of nodes and edges with a " 1 " indicating new edge between the node pairs. If an edge does not exist originally or after link prediction, that entry remains a "0." Table 3 ranks the top muscle atrophy gene-disease associations based on a probability greater than $90 \%$ of link prediction using GNN. The most common disease associated with muscle atrophy are cancer, diabetes, and neural diseases. Table 4 lists the commonly used drugs for these diseases. There are about 180 drugs mentioned in the drug bank database as recommended treatment for the 


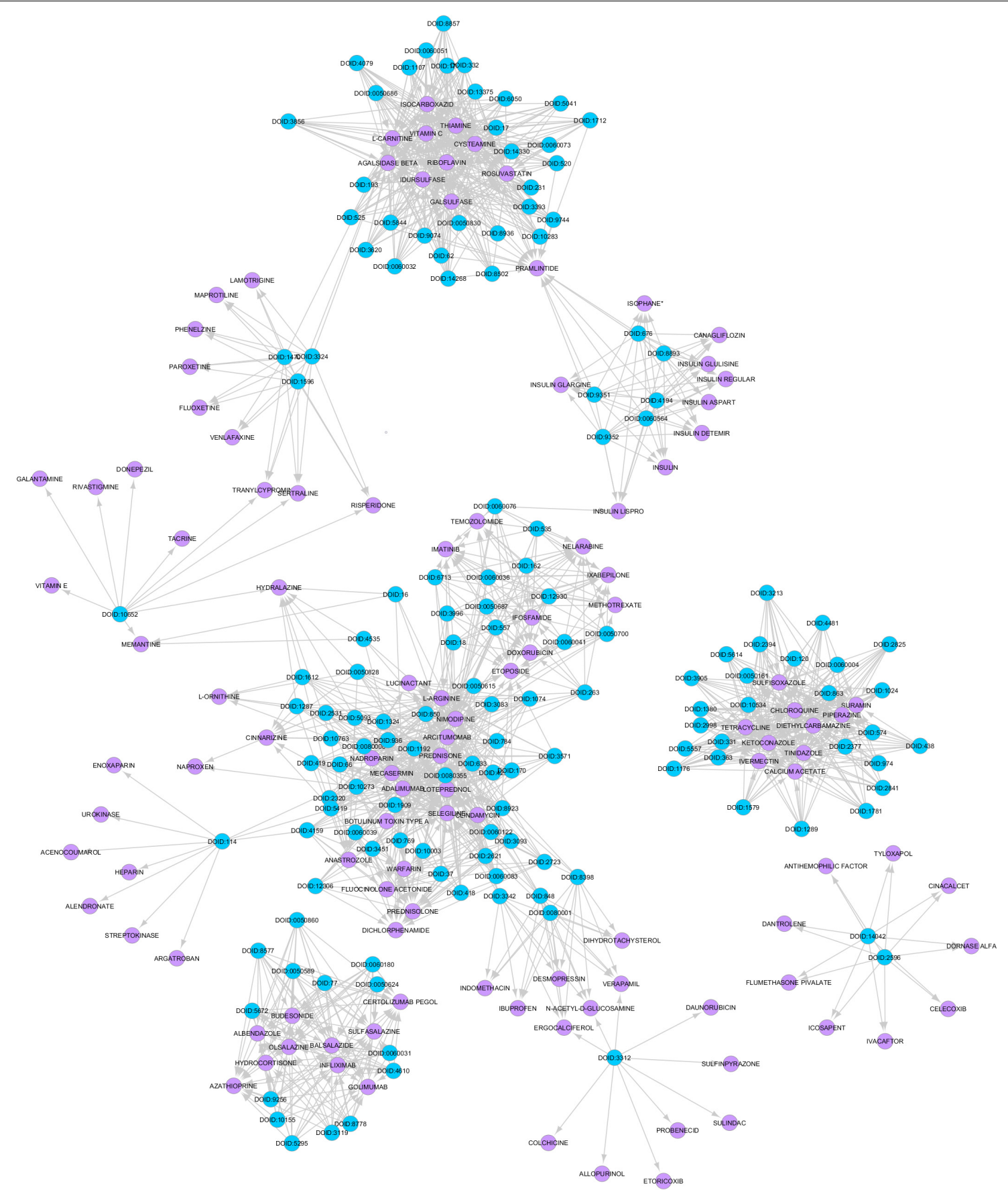

FIGURE 4 | Disease Drug Knowledge Graph constructed from muscle atrophy related diseases and drugs used for their treatment obtained from the drug bank database. The blue colored circles are the disease nodes and the purple colored circles are the drugs.

diseases mentioned in Table 2 which overlap with muscle atrophy condition. Table 5 lists 40 diseases with links to 21 drugs obtained from link prediction with probabilities higher than $80 \%$. Some of these drugs treat more than one disease. Further fewer drugs can be selected by choosing a higher threshold for prediction probability. Table 6 lists the network measures computed for the 21 top ranked drugs in the DDKG. Table 7 lists the network measures for the GDKG and DDKG networks. Table 8 shows the
True Positive, True Negative, False Positive, and False Negative for each of the link prediction methods for the GDKG and DDKG networks. Figures 5, 6 show the ROC curves for the GDKG and the DDKG link prediction, respectively. As can be seen the GNN has higher AUROC, followed by the RF method. The input graph network features are divided into training and validation sets to evaluate the link prediction methods. A 10 -fold cross validation is carried out. Tables 9, 10 summarizes the 10-fold cross validation 
TABLE 2 | Key diseases in which the genes that are maximally regulated in muscle atrophy are involved.

\begin{tabular}{|c|c|c|c|c|}
\hline \multicolumn{5}{|c|}{ Disease Names } \\
\hline Gout & Asthma & Cancer & Adenoma & Colitis \\
\hline Leprosy & Obesity & Alopecia & Glaucoma & Leukemia \\
\hline Lymphoma & Melanoma & Myopathy & Myositis & Rhinitis \\
\hline Syndrome & Vitiligo & Arthritis & Carcinoma & Pemphigus \\
\hline Psoriasis & Tauopathy & Dermatitis & Narcolepsy & Vasculitis \\
\hline Cholangitis & Eye disease & Lung cancer & Scleroderma & Skin cancer \\
\hline Bone disease & Hair disease & Hypertension & Liver cancer & Lung disease \\
\hline Nose disease & Skin disease & Brain disease & Breast cancer & Dental caries \\
\hline Endometriosis & Heart disease & Hypotrichosis & Kidney cancer & Larynx cancer \\
\hline Liver disease & Lymphadenitis & Mood disorder & Mouth disease & Neuroblastoma \\
\hline Overnutrition & Prion disease & Schizophrenia & Skin melanoma & Tooth disease \\
\hline Acute leukemia & Aortic disease & Artery disease & Breast disease & Cardiomyopathy \\
\hline Celiac disease & Hypothyroidism & Kidney disease & Kidney failure & Lung carcinoma \\
\hline Osteoarthritis & Ovarian cancer & Skin carcinoma & Sleep disorder & Spinal disease \\
\hline Stomach cancer & Uterine cancer & B-cell lymphoma & Benign neoplasm & Crohn's disease \\
\hline Genetic disease & Gonadal disease & Graves' disease & Nephrolithiasis & Ovarian disease \\
\hline Prostate cancer & Stomach disease & Synucleinopathy & Thoracic cancer & Uterine disease \\
\hline Allergic disease & Bipolar disorder & Breast carcinoma & Cell type cancer & Kawasaki disease \\
\hline Muscular disease & Pancreas disease & Prostate disease & Thoracic disease & Vascular disease \\
\hline Allergic rhinitis & Bile duct disease & Bronchial disease & Colorectal cancer & Diabetes mellitus \\
\hline Esophageal cancer & Inner ear disease & Intestinal cancer & Laryngeal disease & Leukocyte disease \\
\hline Lymphoid leukemia & Mental depression & Monogenic disease & Nutrition disease & Pancreatic cancer \\
\hline Rheumatic disease & Testicular cancer & Autoimmune disease & Bipolar I disorder & Cognitive disorder \\
\hline Colorectal adenoma & Endometrial cancer & Esophageal disease & Hematologic cancer & Intestinal disease \\
\hline Lymph node disease & Multiple sclerosis & Prostate carcinoma & Psychotic disorder & Sjogren's syndrome \\
\hline Temporal arteritis & Testicular disease & Ulcerative colitis & Alzheimer's disease & Androgenic alopecia \\
\hline Atrial fibrillation & Gallbladder disease & Heart valve disease & Laryngeal carcinoma & Lupus erythematosus \\
\hline Nicotine dependence & open-angle glaucoma & Organ system cancer & Ovarian dysfunction & Parkinson's disease \\
\hline Aortic valve disease & Basal cell carcinoma & Bullous skin disease & Esophageal carcinoma & Immune system cancer \\
\hline Low tension glaucoma & Motor neuron disease & Nasal cavity disease & non-Hodgkin lymphoma & Pancreatic carcinoma \\
\hline Rheumatoid arthritis & Substance dependence & Systemic scleroderma & Thyroid gland cancer & Aortic valve stenosis \\
\hline Biliary tract disease & Demyelinating disease & Disease of metabolism & Endogenous depression & Hepatobiliary disease \\
\hline Immune system disease & Muscle tissue disease & Myocardial infarction & Nervous system cancer & Thyroid gland disease \\
\hline Urinary system cancer & angle-closure glaucoma & Ankylosing spondylitis & Autoimmune thyroiditis & Chronic kidney disease \\
\hline Dilated cardiomyopathy & Endocrine gland cancer & Large intestine cancer & Nasopharyngeal disease & Nervous system disease \\
\hline Sclerosing cholangitis & Sensory system disease & Urinary system disease & Auditory system disease & Cerebrovascular disease \\
\hline Coronary artery disease & Lymphatic system cancer & Squamous cell carcinoma & Thyroid gland carcinoma & Autism spectrum disorder \\
\hline Disease of mental health & Endocrine system disease & Heart conduction disease & Intrinsic cardiomyopathy & Lymphatic system disease \\
\hline Obstructive lung disease & Photosensitivity disease & Type 1 diabetes mellitus & Type 2 diabetes mellitus & Viral infectious disease \\
\hline Autosomal genetic disease & Bone inflammation disease & Cell type benign neoplasm & Connective tissue disease & Creutzfeldt-Jakob disease \\
\hline Major depressive disorder & Neurodegenerative disease & Peripheral artery disease & Polycystic ovary syndrome & Reproductive organ cancer \\
\hline Respiratory system cancer & Teeth hard tissue disease & Acquired metabolic disease & $\begin{array}{c}\text { Autosomal dominant } \\
\text { disease }\end{array}$ & $\begin{array}{c}\text { Glucose metabolism } \\
\text { disease }\end{array}$ \\
\hline Inflammatory bowel disease & Intestinal benign neoplasm & Respiratory system disease & Sensorineural hearing loss & substance-related disorder \\
\hline Disease by infectious agent & $\begin{array}{l}\text { Hepatobiliary system } \\
\text { cancer }\end{array}$ & $\begin{array}{c}\text { Integumentary system } \\
\text { cancer }\end{array}$ & $\begin{array}{c}\text { Reproductive system } \\
\text { disease }\end{array}$ & Testicular germ cell cancer \\
\hline $\begin{array}{l}\text { Acute lymphoblastic } \\
\text { leukemia }\end{array}$ & Bacterial infectious disease & $\begin{array}{c}\text { Chronic lymphocytic } \\
\text { leukemia }\end{array}$ & Disease of anatomical entity & $\begin{array}{l}\text { Hematopoietic system } \\
\text { disease }\end{array}$ \\
\hline $\begin{array}{l}\text { Integumentary system } \\
\text { disease }\end{array}$ & $\begin{array}{l}\text { Organ system benign } \\
\text { neoplasm }\end{array}$ & Plantar fascial fibromatosis & $\begin{array}{l}\text { Systemic lupus } \\
\text { erythematosus }\end{array}$ & $\begin{array}{c}\text { Amyotrophic lateral } \\
\text { sclerosis }\end{array}$ \\
\hline $\begin{array}{l}\text { Cardiovascular system } \\
\text { disease }\end{array}$ & $\begin{array}{c}\text { Central nervous system } \\
\text { cancer }\end{array}$ & Juvenile rheumatoid arthritis & $\begin{array}{l}\text { Marginal zone B-cell } \\
\text { lymphoma }\end{array}$ & $\begin{array}{c}\text { Central nervous system } \\
\text { disease }\end{array}$ \\
\hline $\begin{array}{l}\text { Gastrointestinal system } \\
\text { cancer }\end{array}$ & $\begin{array}{l}\text { Male reproductive organ } \\
\text { cancer }\end{array}$ & $\begin{array}{c}\text { Musculoskeletal system } \\
\text { disease }\end{array}$ & $\begin{array}{c}\text { Primary angle-closure } \\
\text { glaucoma }\end{array}$ & $\begin{array}{c}\text { Carbohydrate metabolism } \\
\text { disease }\end{array}$ \\
\hline
\end{tabular}


TABLE 2 | (Continued)

\begin{tabular}{|c|c|c|c|c|}
\hline \multicolumn{5}{|c|}{ Disease Names } \\
\hline $\begin{array}{l}\text { Gastrointestinal system } \\
\text { disease }\end{array}$ & $\begin{array}{l}\text { Lower respiratory tract } \\
\text { disease }\end{array}$ & $\begin{array}{c}\text { Specific developmental } \\
\text { disorder }\end{array}$ & $\begin{array}{c}\text { Upper respiratory tract } \\
\text { disease }\end{array}$ & $\begin{array}{l}\text { Female reproductive organ } \\
\text { cancer }\end{array}$ \\
\hline $\begin{array}{l}\text { Male reproductive system } \\
\text { disease }\end{array}$ & $\begin{array}{c}\text { Pervasive developmental } \\
\text { disorder }\end{array}$ & $\begin{array}{c}\text { Primary immunodeficiency } \\
\text { disease }\end{array}$ & $\begin{array}{c}\text { Autonomic nervous system } \\
\text { neoplasm }\end{array}$ & $\begin{array}{c}\text { Central nervous system } \\
\text { vasculitis }\end{array}$ \\
\hline $\begin{array}{l}\text { Disease of cellular } \\
\text { proliferation }\end{array}$ & $\begin{array}{l}\text { Laryngeal squamous cell } \\
\text { carcinoma }\end{array}$ & $\begin{array}{c}\text { Peripheral nervous system } \\
\text { disease }\end{array}$ & $\begin{array}{l}\text { Female reproductive } \\
\text { system disease }\end{array}$ & $\begin{array}{c}\text { Peripheral nervous system } \\
\text { neoplasm }\end{array}$ \\
\hline $\begin{array}{l}\text { Abdominal } \\
\text { obesity-metabolic } \\
\text { syndrome }\end{array}$ & $\begin{array}{c}\text { Primary bacterial infectious } \\
\text { disease }\end{array}$ & $\begin{array}{c}\text { Autoimmune disease of } \\
\text { exocrine system }\end{array}$ & $\begin{array}{l}\text { Chronic obstructive } \\
\text { pulmonary disease }\end{array}$ & $\begin{array}{l}\text { Abdominal } \\
\text { obesity-metabolic } \\
\text { syndrome } 1\end{array}$ \\
\hline $\begin{array}{l}\text { Autoimmune disease of } \\
\text { endocrine system }\end{array}$ & $\begin{array}{l}\text { Developmental disorder of } \\
\text { mental health }\end{array}$ & $\begin{array}{c}\text { Gastrointestinal system } \\
\text { benign neoplasm }\end{array}$ & $\begin{array}{c}\text { Attention deficit } \\
\text { hyperactivity disorder }\end{array}$ & $\begin{array}{c}\text { Autoimmune disease of the } \\
\text { nervous system }\end{array}$ \\
\hline $\begin{array}{l}\text { estrogen-receptor negative } \\
\text { breast cancer }\end{array}$ & $\begin{array}{l}\text { Autoimmune disease of } \\
\text { cardiovascular system }\end{array}$ & $\begin{array}{l}\text { Autoimmune disease of } \\
\text { central nervous system }\end{array}$ & $\begin{array}{c}\text { Autoimmune disease of } \\
\text { gastrointestinal tract }\end{array}$ & $\begin{array}{l}\text { Autoimmune disease of } \\
\text { musculoskeletal system }\end{array}$ \\
\hline $\begin{array}{l}\text { Human immunodeficiency } \\
\text { virus infectious disease }\end{array}$ & $\begin{array}{c}\text { Autoimmune disease of } \\
\text { skin and connective tissue }\end{array}$ & & & \\
\hline
\end{tabular}

Identified from the SPOKE GDKG gene-disease network.

accuracies using the link prediction methods for the GDKG and DDKG networks, respectively. The average accuracies obtained for the gene-disease network link predictions are 93.07, 92.32, and $89.72 \%$ for the GNN, RF, and Gboost methods, respectively. The average accuracies obtained for the disease-drug network link predictions are 92.11, 92.63, and 91.62\% for the GNN, RF, and Gboost methods, respectively. Overall, the GNN has the highest accuracy of $92.59 \%$, followed by 92.48 and $90.67 \%$ for the RF and Gboost methods, respectively. The preferential attachment based link prediction gives an average accuracy of 83.92 and $67.06 \%$ for gene disease, and disease-drug link prediction, respectively. Here, we have combined the analysis of the six GeneLab GLDS datasets related to organ muscle atrophy in spaceflight. This is advantageous than analyzing them individually, as it reduces space and time complexity of

TABLE 3 | New muscle atrophy gene disease associations predicted by random walk network measure and GNN.

\begin{tabular}{lcc}
\hline Gene name & Disease name & Link prediction \\
\hline ATF3 & Bone cancer and hypospadias & 15 \\
PTEN & Tumor suppressor & 29 \\
TNFRSF19 & Ovarian cancer & 11 \\
BCL6 & Lymphoma & 8 \\
EEF1B2 & Seizures & 17 \\
UBASH3A & Type 1 diabetes mellitus & 18 \\
ZMIZ1 & Neurodevelopmental disorder & 32 \\
PTPN22 & Type 1 diabetes mellitus & 22 \\
AAMP & Tylosis With Esophageal Cancer & 15 \\
NOTCH1 & leukemia & 28 \\
CDKN1B & Cell type cancer & 8 \\
FAM167A & Maturity-onset diabetes & 11 \\
DNMT3B & Immunodeficiency & 15 \\
ATF7IP & Testicular germ cell cancer & 14 \\
FADS1 & Lipid metabolism disorder & 18 \\
\hline
\end{tabular}

1 indicates existing association, 0 indicates predicted links. processing. The three methods of RF, Gradient boosting, and GNN perform equally well, while the GNN shows a slightly higher accuracy.

\section{DISCUSSION}

The shared key genes from the Markov Blanket GRN of all the six GeneLab datasets with maximal differential regulation are given in Table 1. Figure 3 shows the GDKG constructed using the top regulated genes from the six GeneLab datasets and the SPOKE database. The red nodes represent the genes, and the blue nodes represent diseases. Table 2 lists the disease nodes present in Figure 3. Table 3 lists 15 new gene disease associations predicted by the GNN link prediction method. There are several differentially regulated genes resulting in reduced proliferation of thymic cells, thereby reducing the size of the thymus (Horie et al., 2019a). Of these the ATF3 is a key gene player identified in Table 1. This gene encodes a member of the mammalian activation transcription factor and is induced by a variety of signals, including many of those encountered by cancer cells. It is involved in the complex process of cellular stress response. This gene has 15 additional links predicted by the GNN. PTEN is an important gene that suppresses cell growth into tumors, which has been identified as a key gene in the GDKG. This gene is found to regulate muscle protein degradation in diabetes ( $\mathrm{Hu}$ et al., 2007). In the GDKG network this gene has 33 existing links, and 29 new links to existing diseases are predicted. Tumor Necrosis Factor (TNF) is one of the most important musclewasting cytokine, elevated levels of which cause significant muscular abnormalities (Bhatnagar et al., 2010). The protein encoded by TNFRSF19 is a member of the TNF-receptor family. When overexpressed it activates the JNK signaling pathway. The diseases associated with this gene are ovarian cancer and ectodermal dysplasia (Dostert et al., 2019). This gene originally had nine links in the GDKG, and eleven new links were added by the GNN link prediction method implying the importance of this gene in muscle atrophy prognosis in spaceflight. The 
TABLE 4 | Significant drugs used for treatment of diseases listed in Table 2.

\begin{tabular}{|c|c|c|c|c|}
\hline \multicolumn{5}{|c|}{ Drugs } \\
\hline Doxorubicin & Risperidone & Ivermectin & Rivastigmine & Streptokinase \\
\hline Arcitumomab & Sertraline & Piperazine & Diethylcarbamazine & Cinnarizine \\
\hline Nelarabine & Paroxetine & Suramin & Aminoglutethimide & Argatroban \\
\hline Ifosfamide & Lamotrigine & Selegiline & Prednisolone & Nadroparin \\
\hline Ixabepilone & Phenelzine & Albendazole & Certolizumab pegol & Lucinactant \\
\hline Imatinib & Venlafaxine & Budesonide & Dichlorphenamide & Clindamycin \\
\hline Etoposide & Isocarboxazid & Olsalazine & $\begin{array}{l}\text { Fluocinolone } \\
\text { acetonide }\end{array}$ & Telithromycin \\
\hline Nimodipine & L-carnitine & Balsalazide & Loteprednol & Melatonin \\
\hline Temozolomide & Thiamine & Verapamil & $\begin{array}{l}\text { Antihemophilic } \\
\text { factor }\end{array}$ & Amphetamine \\
\hline Methotrexate & Galsulfase & Sulfasalazine & Anastrozole & Citalopram \\
\hline Canagliflozin & Idursulfase & Warfarin & Acenocoumarol & Amisulpride \\
\hline Insulin regular & Rosuvastatin & Azathioprine & Hydrocortisone & Fluvoxamine \\
\hline Insulin lispro & Cysteamine & Infliximab & $\begin{array}{l}\mathrm{N} \text {-acetyl-d- } \\
\text { glucosamine }\end{array}$ & Sulfapyridine \\
\hline Insulin aspart & Icosapent & Golimumab & Indomethacin & Naproxen \\
\hline Insulin glargine & Vitamin c & Adalimumab & Dihydrotachysterol & Tretinoin \\
\hline $\begin{array}{l}\text { Insulin, } \\
\text { isophane }\end{array}$ & Riboflavin & L-arginine & Ergocalciferol & Ibuprofen \\
\hline $\begin{array}{l}\text { Insulin } \\
\text { glulisine }\end{array}$ & Secretin & Hydralazine & $\begin{array}{l}\text { Botulinum toxin } \\
\text { type a }\end{array}$ & Mecasermin \\
\hline Insulin detemir & Ketoconazole & Memantine & Desmopressin & Isopropamide \\
\hline Pramlintide & Sulfisoxazole & Galantamine & $\begin{array}{l}\text { Nandrolone } \\
\text { decanoate }\end{array}$ & Inulin \\
\hline L-carnitine & Tinidazole & Donepezil & $\begin{array}{l}\text { Nandrolone } \\
\text { phenpro. }\end{array}$ & Heparin \\
\hline Secretin & Chloroquine & Tacrine & $\begin{array}{l}\text { Glucagon } \\
\text { recombinant }\end{array}$ & Mifepristone \\
\hline Fluoxetine & Tetracycline & Vitamin e & $\begin{array}{l}\text { Nandrolone } \\
\text { phenpro. }\end{array}$ & Diazoxide \\
\hline Insulin lispro & Icosapent & Daunorubicin & Sulfinpyrazone & Colchicine \\
\hline Allopurinol & Sulindac & Etoricoxib & $\begin{array}{l}\text { Flumethasone } \\
\text { pivalate }\end{array}$ & Dronedarone \\
\hline Cinacalcet & Dantrolene & Celecoxib & $\begin{array}{l}\text { Antihemophilic } \\
\text { factor }\end{array}$ & Tyloxapol \\
\hline Maprotiline & Icosapent & Dornase alfa & $\begin{array}{l}\text { Nandrolone } \\
\text { decanoate }\end{array}$ & Urokinase \\
\hline Insulin lispro & L-ornithine & Daunorubicin & Sulfinpyrazone & Colchicine \\
\hline Probenecid & Allopurinol & Sulindac & Etoricoxib & Naproxen \\
\hline Dronedarone & Secretin & Cinacalcet & Dantrolene & Celecoxib \\
\hline Prednisone & Tyloxapol & Ivacaftor & Agalsidase beta & Dornase alfa \\
\hline Enoxaparin & Alendronate & Probenecid & Calcium acetate & Ivacaftor \\
\hline Insulin lispro & L-ornithine & Daunorubicin & Sulfinpyrazone & Colchicine \\
\hline Probenecid & Allopurinol & Sulindac & Etoricoxib & Naproxen \\
\hline Dronedarone & Secretin & Cinacalcet & Dantrolene & Celecoxib \\
\hline Prednisone & Tyloxapol & Ivacaftor & Tranylcypromine & Dornase alfa \\
\hline Enoxaparin & Alendronate & Probenecid & Aminoglutethimide & Ivacaftor \\
\hline
\end{tabular}

BCL6 gene is a regulator of T-cell-dependent inflammation and autoimmune responses. BCL6 is likely to regulate B and T-cells via cell-specific biochemical mechanisms. Dysregulation of BCL6 could contribute to BCL6+ T-cell lymphomas and regulated in urinary bladder urothelial carcinoma (Wu et al., 2020). This gene has eight existing links and eight links have been added by the link prediction method, showing the importance of this
TABLE 5 | Possible drugs for repurposing for muscle atrophy treatment predicted by random walk network feature and GNN.

\begin{tabular}{|c|c|c|}
\hline Disease name & Repurposed drugs & Probabilities \\
\hline Integumentory system cancer & Arcitumomab & 90.52 \\
\hline Hypertension & L-arginine & 89.44 \\
\hline Type 2 diabetes mellitus & Insulin & 88.980385 \\
\hline Cardiovascular system disease & Selegiline & 87.93291 \\
\hline Ulcerative colitis & Infliximab & 87.20253 \\
\hline Autonomic nervous system neoplasm & Loteprednol & 87.038925 \\
\hline Hematologic cancer & L-ornithine & 86.95308 \\
\hline Ulcerative colitis & Olsalazine & 86.79947 \\
\hline Vitiligo & Loteprednol & 86.54014 \\
\hline Crohn's disease & Infliximab & 86.485664 \\
\hline Gastrointestinal system cancer & Golimumab & 86.456894 \\
\hline Colitis & Olsalazine & 86.39726 \\
\hline Colitis & Azathioprine & 86.141464 \\
\hline Intestinal benign neoplasm & Olsalazine & 86.11937 \\
\hline Inflammatory bowel disease & Hydrocortisone & 86.08773 \\
\hline Crohn's disease & Olsalazine & 85.965416 \\
\hline Intestinal cancer & Olsalazine & 85.94351 \\
\hline Crohn's disease & Balsalazide & 85.63084 \\
\hline Colorectal cancer & Hydrocortisone & 85.55948 \\
\hline Systemic scleroderma & L-arginine & 85.43495 \\
\hline Crohn's disease & Certolizumab pegol & 85.31858 \\
\hline Gastrointestinal system disease & Balsalazide & 85.06852 \\
\hline Dermatitis & L-ornithine & 84.991 \\
\hline Gastrointestinal system disease & Certolizumab pegol & 84.908 \\
\hline Gastrointestinal system benign neoplasm & Balsalazide & 84.827 \\
\hline Crohn's disease & Budesonide & 84.744 \\
\hline Muscular disease & L-ornithine & 84.532 \\
\hline Demyelinating disease & Tinidazole & 83.901 \\
\hline Demyelinating disease & Ivermectin & 83.811 \\
\hline Leprosy & Tetracycline & 83.778 \\
\hline Peripheral artery disease & Riboflavin & 83.776 \\
\hline Nasal disorder & Tetracycline & 83.659 \\
\hline Muscular disease & Adalimumab & 83.642 \\
\hline Reproductive organ cancer & Tetracycline & 83.485 \\
\hline Hematologic cancer & Nimodipine & 83.203 \\
\hline Autoimmune disease of the nervous system & Ivermectin & 83.107 \\
\hline Testicular cancer & Tinidazole & 82.969 \\
\hline Testicular cancer & Sulfisoxazole & 82.877 \\
\hline Neurodegenerative disease & Tinidazole & 82.860 \\
\hline Neurodegenerative disease & Tetracycline & 82.828 \\
\hline
\end{tabular}

gene in spaceflight induced muscle atrophy. The EEF1B2 gene encodes a translation elongation factor specifically expressed in neurons and muscles (Doig et al., 2013). The protein is a guanine nucleotide exchange factor involved in the transfer of aminoacylated tRNAs to the ribosome. Diseases associated with EEF1B2 are seizures, alacrima, achalasia, and intellectual instability syndrome. This gene has seven existing links in the GDKG, and 17 new predicted links. Apart from these five key genes there are 10 more mentioned in Table 3. The network measures for these 15 genes are listed in Table 1. Compared to 
TABLE 6 | Network measures for possible drug treatments for muscle atrophy in spaceflight.

\begin{tabular}{|c|c|c|c|}
\hline Drug name & $\begin{array}{c}\text { Degree } \\
\text { distribution }\end{array}$ & $\begin{array}{l}\text { Neighborhood } \\
\text { connectivity }\end{array}$ & $\begin{array}{l}\text { Subgraph } \\
\text { centrality }\end{array}$ \\
\hline Adalimumab & 42 & 9.76 & $437,551.8$ \\
\hline Arcitumomab & 60 & 9.83 & $652,802.9$ \\
\hline Azathioprine & 14 & 10 & $6,881.267$ \\
\hline Balsalazide & 14 & 10 & $6,881.267$ \\
\hline Budesonide & 14 & 10 & $6,881.267$ \\
\hline Certolizumab pegol & 14 & 10 & $6,881.267$ \\
\hline Golimumab & 14 & 10 & $6,881.267$ \\
\hline Hydrocortisone & 14 & 10 & $6,881.267$ \\
\hline Infliximab & 14 & 10 & $6,881.267$ \\
\hline Insulin & 6 & 10 & 39.17687 \\
\hline Ivermectin & 26 & 10 & $5,830.759$ \\
\hline L-arginine & 30 & 10 & $258,302.4$ \\
\hline L-ornithine & 5 & 10 & $79,395.46$ \\
\hline Loteprednol & 40 & 10 & $469,331.1$ \\
\hline Nimodipine & 48 & 9.79 & $402,801.8$ \\
\hline Olsalazine & 14 & 10 & $6,881.267$ \\
\hline Riboflavin & 31 & 10.45 & $79,395.46$ \\
\hline Selegiline & 45 & 10 & $523,266.8$ \\
\hline Sulfisoxazole & 26 & 10 & $138,229.6$ \\
\hline Tetracycline & 26 & 10 & $138,229.6$ \\
\hline Tinidazole & 26 & 10 & $138,229.6$ \\
\hline
\end{tabular}

TABLE 7 | Network measures for the GDKG and DDKG networks.

\begin{tabular}{lcc}
\hline Network measure & GDKG & DDKG \\
\hline Spectral gap & 9.015 & 1.011 \\
Density & 0.027 & 0.048 \\
Average number of neighbors & 7.993 & 11.178 \\
\hline
\end{tabular}

the other genes in Table 1, these 15 genes with higher number of predicted links also have higher values of degree distribution, neighborhood connectivity, and subgraph centrality network measures, as listed in Table 1. These genes also have higher link prediction probabilities greater than $90 \%$. The diseases associated with these genes are cancer, diabetes, and neurological disorders most of which have muscle atrophy as a side effect. Prolonged exposure to spaceflight may cause risk of contracting these diseases. Hence, preventive medicine and therapeutics are key in warding off these conditions.

\section{Implications for Spaceflight}

Several spaceflight experiments have shown that changes in the physical environment modulate cellular responses thus accelerating the risk of age-related diseases such as bone loss, muscle atrophy, and impaired immune responses (Versari et al., 2013; Cadena et al., 2019). Investigations on muscle atrophy in organs and tissues including cutaneous muscles in rodent and human models are being conducted in spaceflight for over a decade (Däpp et al., 2004; Neutelings et al., 2015; Goropashnaya et al., 2020). There are about 20 datasets available in GeneLab on muscle atrophy investigation on animal
TABLE 8 | Results using Graph Neural Network (GNN), Gradient Boosting (GB), and Random Forest (RF) for link prediction in the Gene Disease Knowledge Graph (GDKG) and Disease Drug Knowledge Graph (DDKG).

\begin{tabular}{lccccccc}
\hline & \multicolumn{3}{c}{ GDKG } & & & \multicolumn{3}{c}{ DDKG } \\
\cline { 3 - 4 } & GNN & RF & GB & & GNN & RF & GB \\
\hline TP & 448 & 476 & 466 & 235 & 254 & 249 \\
TN & 196 & 159 & 145 & 272 & 262 & 262 \\
FP & 34 & 71 & 85 & 9 & 21 & 21 \\
FN & 56 & 28 & 38 & 47 & 40 & 45 \\
\hline
\end{tabular}

Table shows number of True Positive (TP), True Negative (TN), False Positive (FP), and False Negative (FN) rates.

models in spaceflight (NASA Gene Lab data repository, n.d.). Formeterol is the only drug tested so far in spaceflight to mitigate muscle atrophy in mice (Ballerini et al., 2020). While experimental drug repurposing and clinical testing are prolonged and expensive, our proposed network science and artificial intelligence framework is computationally inexpensive and can be used for the rapid selection of candidate drugs to treat muscle atrophy in spaceflight. As muscle atrophy is a condition caused by many terrestrial diseases, the medications prescribed for these diseases can be useful candidates for repurposing for muscle atrophy. Hence, we constructed the GDKG for muscle atrophy to determine the diseases that have muscle atrophy as a primary side effect, and performed link prediction to identify the drugs that treat these diseases and can be repurposed for treating muscle atrophy. Figure 4 shows the DDKG constructed from the top ranked gene diseases associations from the GDKG, and the drugs used in treating these diseases. The blue nodes represent diseases, and the purple nodes represent the drugs. Table 4 lists the drugs from the network in Figure 4. The three link prediction algorithms are applied to the DDKG for identifying possible drugs for muscle atrophy treatment. Table 5 lists the drugs with probabilities higher than $80 \%$. These drugs are used for treating the conditions that have muscle atrophy as a severe side effect such as cancer, diabetes, and nervous system disorders. For example, antidiabetic agents such as metformin, incretins, vitamin $\mathrm{D}$, formoterol are medications that can reduce muscle wastage while treating diabetes (Campins et al., 2017). Indeed the GeneLab datasets GLDS-244 and GLDS-245 were collected to evaluate the efficacy of the drug formoterol to treat muscle atrophy in spaceflight flown mice (Ballerini et al., 2020). Muscle loss is also present in Chronic Obstructive Pulmonary Disease (COPD). The medication bimagrumab that treats COPD also resulted in increase in thigh muscle volume. By constructing the DDKG and applying link prediction, we have identified drugs belonging to the Monoclonal AntiBodies (MABs) family that are used for treating cancer as promising candidates for muscle atrophy in spaceflight. These include adalimumab, arcitumomab, certolizumab, golimumab, and infliximab. Table 5 lists the probabilities for these drugs as well as others that treat cancer and other diseases. Hence, one drug is repeated several times in Table 5. In total, there are 21 drugs that have higher probabilities for predicted links. The network measures for these drug nodes in the DDKG network is listed 


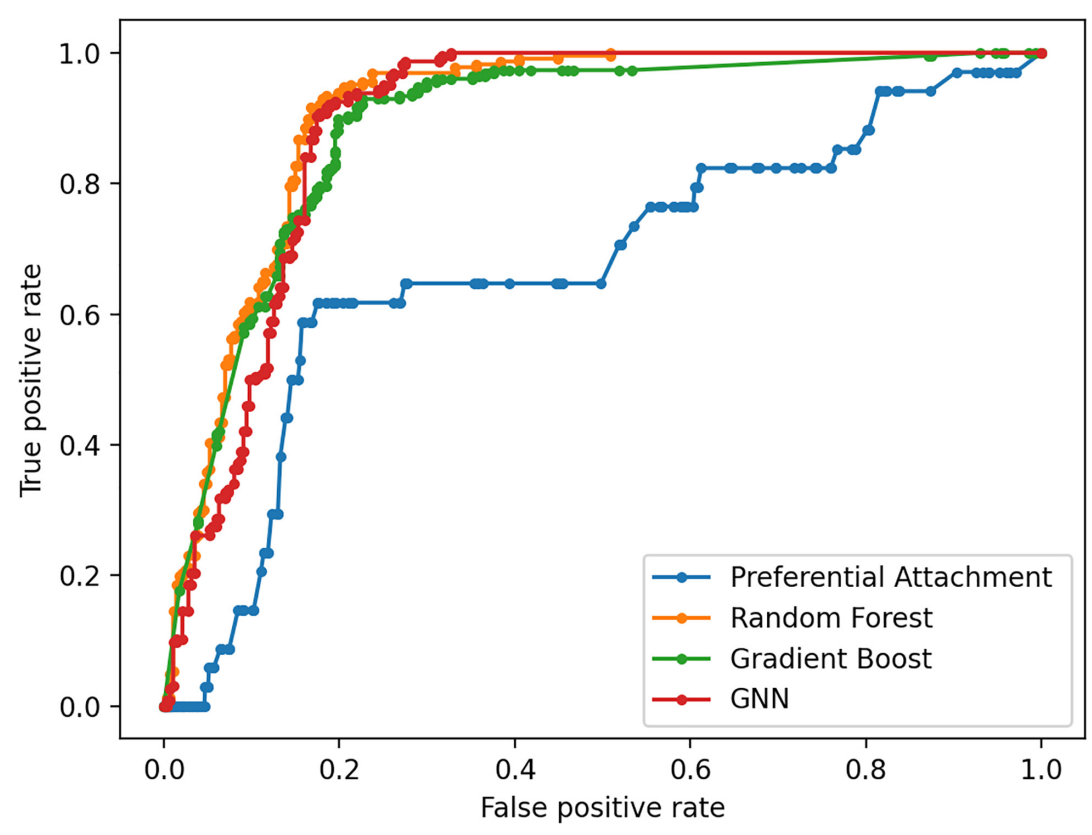

FIGURE 5 | Receiver Operating Characteristic curves for link prediction between genes differentially regulated in muscle atrophy and diseases in the GDKG using PA, RF, Gboost, and GNN methods.

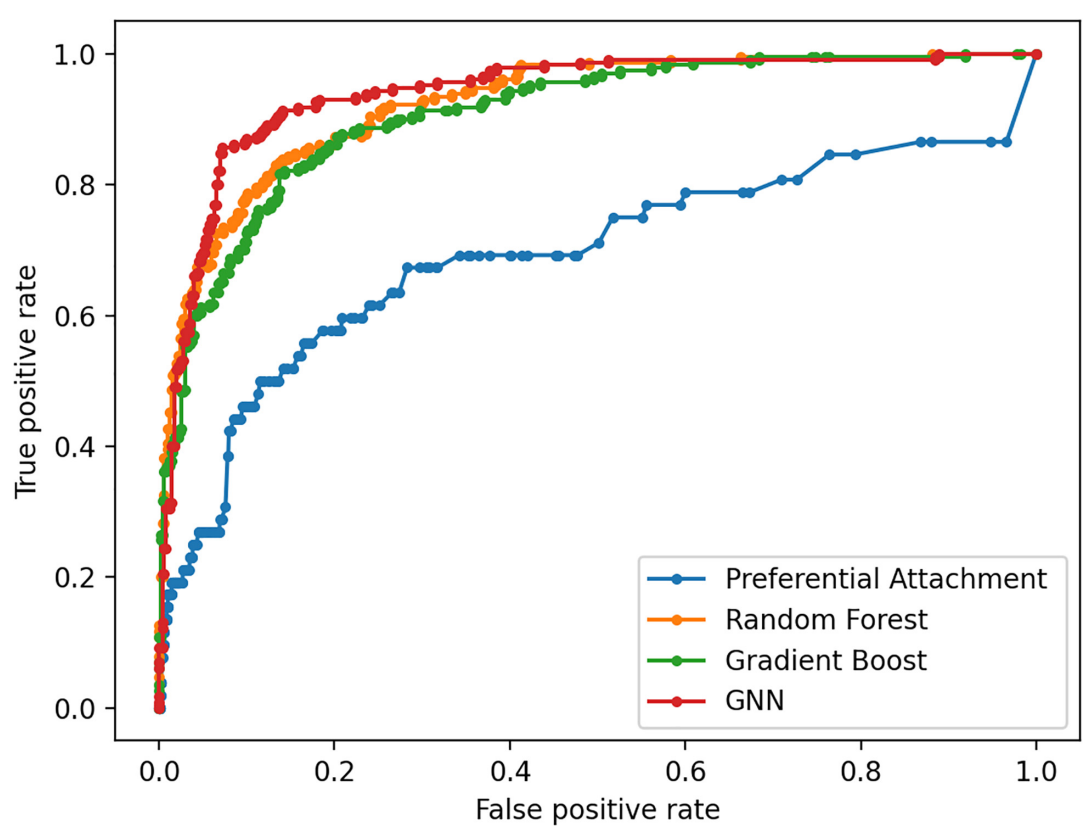

FIGURE 6 | Receiver Operating Characteristic curves for link prediction between muscle atrophy related diseases and drugs used for their treatment in the DDKG using PA, RF, Gboost and GNN methods.

TABLE 9 | Ten-fold cross validation accuracies for link prediction using RF, Gboost, and GNN in GDKG.

\begin{tabular}{lccccccccccc}
\hline Methods & $\mathbf{1}$ & $\mathbf{2}$ & $\mathbf{3}$ & $\mathbf{4}$ & $\mathbf{5}$ & $\mathbf{6}$ & $\mathbf{7}$ & $\mathbf{8}$ & $\mathbf{9}$ & $\mathbf{1 0}$ & AUROC \\
\hline RF & 92.17 & 93.65 & 95.91 & 92.66 & 91.55 & 96.59 & 91.13 & 92.57 & 92.49 & 90.55 & 92.32 \\
Gboost & 87.86 & 90.56 & 89.32 & 88.78 & 88.25 & 93.19 & 86.07 & 90.55 & 88.34 & 88.75 & 89.72 \\
GNN & 95.79 & 95.20 & 96.38 & 89.58 & 94.50 & 95.86 & 96.44 & 95.30 & 94.60 & 91.69 & 93.07
\end{tabular}


TABLE 10 | Ten-fold cross validation accuracies for link prediction using RF, Gboost, and GNN in DDKG.

\begin{tabular}{|c|c|c|c|c|c|c|c|c|c|c|c|}
\hline Methods & 1 & 2 & 3 & 4 & 5 & 6 & 7 & 8 & 9 & 10 & AUROC \\
\hline $\mathrm{RF}$ & 91.20 & 86.69 & 88.77 & 93.09 & 88.76 & 93.55 & 89.48 & 92.74 & 86.13 & 88.30 & 92.63 \\
\hline Gboost & 91.49 & 89.54 & 91.46 & 91.78 & 89.70 & 94.23 & 90.09 & 93.58 & 88.74 & 89.68 & 91.62 \\
\hline GNN & 88.15 & 87.80 & 88.67 & 92.00 & 88.81 & 90.91 & 91.01 & 89.11 & 87.39 & 86.95 & 92.11 \\
\hline
\end{tabular}

in Table 6. As can be seen, all of these drugs have similar values for degree distribution, and have a neighborhood connectivity between 9 and 10. The drugs with highest measures for degree distribution, neighborhood connectivity, and subgraph centrality are Nimodipine, Arcitumomab, Selegiline, Tetracydine, and Loteprednol. Arcitumomab, L-Arginine, L-Ornithine, and Nimodipine which are used for treating cancer and muscle disuse. Selgiline is used for treating cardiovascular diseases. Most of the 21 drugs that can be repurposed for muscle atrophy treat some type of cancer. The repurposing of a drug to treat muscle atrophy is limited by the drug database as the condition itself is secondary to diseases that have no cures. The selection of drugs to treat muscle atrophy in spaceflight could be based on those that can provide clear cures and can be effectively repurposed.

\section{Network Analysis}

Table 7 lists the network measures of girth, density and spectral gap for the GDKG and DDKG networks. As can be seen from these measures the GDKG network has higher spectral gap of 9.015. The larger the spectral gap (the smaller $\left|\lambda_{2}\right|$ ), the higher the network flow with sparseness, expansion, diffusion, and random walk. Hence, these networks have a higher measure of random walks, implying that the nodes that lie closer to each other in the network perform similar functions. The advantage of using networks and AI methods for drug repurposing is that the graphs themselves are scalable and can include more genes, disease, and drug nodes and the deep learning architecture can be built to handle corresponding large scale prediction problems. The network sciences approach and the AI based tool can be used to predict key targets and potential diseases arising from spaceflight missions and will facilitate countermeasure development.

\section{Key Genes Description}

Table 1 lists the highly activated genes from the spaceflight mice muscle atrophy datasets. These genes are involved in protein amino acid binding, glycoprotein binding, cell growth and/or maintenance, and cell adhesion receptor inhibitor activity. These genes are part of cellular metabolic pathways by which individual cells transform chemical substances and pathways involving organic or inorganic compounds that contain nitrogen. They are also involved in chemical reactions and pathways involving an organic substance, any molecular entity containing carbon, and in chemical reactions and pathways involving those compounds which are formed as a part of the normal anabolic and catabolic processes. Some of these genes are involved in organ system process carried out by any of the organs or tissues of the neurological system. $15 \mathrm{key}$ genes with the highest number of newly predicted links is given in Table 3 and their associated diseases from diseases from GeneCards (2021) is also given here.
As can be seen half of these genes are associated with some type of cancer, followed by diabetes.

\section{CONCLUSION}

We have presented a novel method for generating GDKGs for a particular disease from gene expression datasets using network analysis and the SPOKE database. In this research, we have worked with transcriptional gene expression datasets for muscle atrophy in mice flown in spaceflight microgravity. Link prediction applied to this network reveals interesting relationships of key genes with different types of cancer. The link prediction method is also used on the Disease Drug Knowledge Graph resulting in the identification of novel drugs that are possible candidates for treating muscle atrophy accelerated due to spaceflight travel. We have combined six GeneLab datasets in an innovative way with disease and drug databases and applied network analysis and artificial intelligence methods for drug repurposing.

\section{DATA AVAILABILITY STATEMENT}

Publicly available datasets were analyzed in this study. This data can be found here: genelab.nasa.gov.

\section{AUTHOR CONTRIBUTIONS}

VM: conceptualization, supervision, project administration, and funding acquisition. VM, JO-S, and VD-M: methodology and formal analysis. JO-S and VD-M: software, validation, data curation, and visualization. $\mathrm{VM}$ and JO-S: investigation and writing-original draft preparation, review, and editing. All authors have read and agreed to the published version of the manuscript.

\section{FUNDING}

This research was funded by NASA EPSCoR (Grant Number 80NSSC20M0132). Opinions, findings, conclusions, or recommendations expressed in this material are those of the authors and do not necessarily reflect the views of NASA.

\section{ACKNOWLEDGMENTS}

The authors would like to thank the NASA technical monitor Egle Cekanaviciute for providing us with the SPOKE database for gene disease interactions. 


\section{REFERENCES}

Abbas, K., Abbasi, A., Dong, S., Niu, L., Yu, L., Chen, B., et al. (2021). Application of network link prediction in drug discovery BMC Bioinform. 22:187. doi: 10.1186/s12859-021-04082-y

Ainsworth, H., Shin, S., and Cordell, H. (2017). A comparison of methods for inferring causal relationships between genotype and phenotype using additional biological measurements. Genet. Epidemiol. 41, 577-586. doi: 10.1002/gepi. 22061

Anand, G. K. (2009). Bioinformatics Bayesian Networks for Omics Data Analysis. Wageningen, The Netherlands: Thesis Wageningen University.

Ballerini, A., Chua, C., Rhudy, J., Susnjar, A., Di Trani, N., Jain, P., et al. (2020). Counteracting muscle atrophy on earth and in space via nanofluidics delivery of formoterol. Adv. Therapeutics 3:2000014. doi: 10.1002/adtp.202000014

Beheshti, A., Ray, S., Fogle, H., Berrios, D., and Costes, S. (2018). A microRNA signature and TGF- $\beta 1$ response were identified as the key master regulators for spaceflight response. PLoS One 13:e0199621. doi: 10.1371/journal.pone. 0199621

Bei, Y., and Xiao, J. (2017). MicroRNAs in muscle wasting and cachexia induced by heart failure. Nat. Rev. Cardiol. 14:566. doi: 10.1038/nrcardio.2017.122

Bhatnagar, S., Panguluri, S., Gupta, S., Dahiya, S., Lundy, R., et al. (2010). Tumor necrosis Factor- $\alpha$ regulates distinct molecular pathways and gene networks in cultured skeletal muscle cells. PLoS One 5:e13262. doi: 10.1371/journal.pone. 0013262

Biggs, N. (1993). Algebraic Graph Theory. 2nd edn. Cambridge: Cambridge University Press.

Bui, A., and Jun, H. (2012). Learning bayesian network structure using Markov blanket decomposition. Pattern Recogn. Lett. 33, 2134-2140. doi: 10.1016/j. patrec.2012.06.013

Burckart, K., Beca, S., Urban, R., and Sheffield-Moore, M. (2010). Pathogenesis of muscle wasting in cancer cachexia: targeted anabolic and anticatabolic therapies. Curr. Opin. Clin. Nutr. Metab. Care 13, 410-416. doi: 10.1097/mco. 0b013e328339fdd2

Cadena, S., Zhang, Y., Fang, J., Brachat, S., Kuss, P., Giorgetti, E., et al. (2019). Skeletal muscle in MuRF1 null mice is not spared in low-gravity conditions, indicating atrophy proceeds by unique mechanisms in space. Sci. Rep. 9:9397. doi: 10.1038/s41598-019-45821-9

Campins, L., Camps, M., Riera, A., Pleguezuelos, E., Yebenes, J., et al. (2017). Oral drugs related with muscle wasting and sarcopenia. a review. Pharmacology 99, 1-8. doi: 10.1159/000448247

Chen, B., Yong, H., and Ying, J. (2018). Link prediction on directed networks based on AUC optimization. IEEE Access 6, 28122-28136. doi: 10.1109/access.2018. 2838259

Chen, Z., Liu, X., Hogan, W., Shenkman, E., and Bian, J. (2020). Applications of artificial intelligence in drug development using real-world data. Drug Discovery Today 26, 1256-1264.

Costa, L., and Travieso, G. (2007). Exploring complex networks through random walks. Phys. Rev. Statistical Nonlinear Soft Matter Phys. 75, 1-7.

Däpp, C., Flück, M., Schmutz, S., and Hoppeler, H. (2004). Transcriptional reprogramming and ultrastructure during atrophy and recovery of mouse soleus muscle. Physiol. Genom. 20, 97-107. doi: 10.1152/physiolgenomics. 00100.2004

Doig, J., Griffiths, L., Peberdy, D., Dharmasaroja, P., Vera, M., Davies, F., et al. (2013). In vivo characterization of the role of tissue-specific translation elongation factor $1 \mathrm{~A} 2$ in protein synthesis reveals insights into muscle atrophy. FEBS J. 280: 6528-6540. doi: 10.1111/febs.12554

Dostert, C., Grusdat, M., Letellier, E., and Brenner, D. (2019). The TNF family of ligands and receptors: communication modules in the immune system and beyond. Physiol. Rev. 99, 115-160. doi: 10.1152/physrev.00045. 2017

Dudgeon, W., Phillips, K., Carson, J., Brewer, R., Durstine, J., et al. (2006). Counteracting muscle wasting in HIV-infected individuals. HIV Med. 7, 299310. doi: 10.1111/j.1468-1293.2006.00380.x

Fire, M., Tenenboim, L., Lesser, O., Puzis, R., Rokach, L., and Elovici, Y., (2011). "Link prediction in social networks using computationally efficient topological features," In Proceedings - 2011 IEEE International Conference on Privacy, Security, Risk and Trust and IEEE International Conference on Social Computing, PASSAT/SocialCom 2011 (Piscataway, NJ: IEEE): 73-80.
GeneCards (2021). DrugBank Online. Database for Drug and Drug Target Info. [online]. Available online at: Go.drugbank.com (accessed September 1, 2020).

Goropashnaya, A., Barnes, B., and Fedorov, V. (2020). Transcriptional changes in muscle of hibernating arctic ground squirrels (Urocitellus parryii): implications for attenuation of disuse muscle atrophy. Sci. Rep. 10:9010.

Grover, A., and Leskovec, J. (2016). "node2vec: scalable feature learning for networks," in Proceedings of the ACM SIGKDD International Conference on Knowledge Discovery and Data Mining (New York, NY: ACM), $855-864$.

Gysi, D., Valle, I., Zitnik, M., Ameli, A., Gan, X., Varol, O., et al. (2020). Network Medicine Framework for Identifying Drug Repurposing Opportunities for COVID-19. ArXiv [preprint]. https://www.ncbi.nlm.nih.gov/pmc/articles/ PMC7280907/ (accession April 15, 2021). .

Horie, K., Kato, T., Kudo, T., Sasanuma, H., Miyauchi, M., Akiyama, N., et al. (2019a). Impact of spaceflight on the murine thymus and mitigation by exposure to artificial gravity during spaceflight. Sci. Rep. 9:19866.

Horie, K., Sasanuma, H., Kudo, T., Fujita, S., Miyauchi, M., Miyao, T., et al. (2019b). Down-regulation of GATA1-dependent erythrocyte-related genes in the spleens of mice exposed to a space travel. Sci. Rep. 9:7654.

Hu, Z., Lee, I., Wang, X., Sheng, H., Zhang, L., Du, J., et al. (2007). PTEN expression contributes to the regulation of muscle protein degradation in diabetes. Diabetes Metab. Res. Rev. 56, 2449-2456. doi: 10.2337/db06-1731

Hussain Ahmed, C., Dhruba Kumar, B., and Jugal Kumar, K. (2020). (Differential) Co-expression analysis of gene expression: a survey of best practices. IEEE/ACM Trans. Comp. Biol. Bioinform. 17, 1154-1173.

Janwa, H., Massey, S., Velev, J., and Mishra, B. (2019). On the origin of biomolecular networks. Front. Genetics 10:240. doi: 10.3389/fgene.2019.00240

Jarada, T., Rokne, J., and Alhajj, R. (2020). A review of computational drug repositioning: strategies, approaches, opportunities, challenges, and directions. J. Cheminform. 12:46.

Kalyani, R., Corriere, M., and Ferrucci, L. (2014). Age-related and disease-related muscle loss: the effect of diabetes, obesity, and other diseases. Lancet Diabetes Endocrinol. 2, 819-829. doi: 10.1016/s2213-8587(14)70034-8

Koromina, M., Pandi, M., and Patrinos, G. (2019). Rethinking drug repositioning and development with artificial intelligence, machine learning, and omics. OMICS J. Int. Biol. 23, 539-548. doi: 10.1089/omi.2019.0151

Lebsack, T., Fa, V., Woods, C., Gruener, R., Manziello, A., Pecaut, M., et al. (2010). Microarray analysis of spaceflown murine thymus tissue reveals changes in gene expression regulating stress and glucocorticoid receptors. J. Cell. Biochem. 110:372-381

Li, T., Wang, J., Tu, M., Zhang, Y., and Yan, Y. (2016). Enhancing link prediction using gradient boosting features. Lecture Notes Comp. Sci. 9772, 81-92. doi: 10.1007/978-3-319-42294-7_7

Malkani, S., Chin, C., Cekanaviciute, E., Mortreux, M., Okinula, H., Tarbier, M., et al. (2020). Circulating miRNA spaceflight signature reveals targets for countermeasure development. Cell Rep. 33:108448. doi: 10.1016/j.celrep.2020. 108448

Muscular Dystrophy (n.d.). Available online at: https://www.mayoclinic.org/ diseasesconditions/muscular-dystrophy/symptoms-causes/syc-20375388

Mutlu, E., Oghaz, T., Rajabi, A., and Garibay, I. (2020). Review on learning and extracting graph features for link prediction. Machine Learn. Knowledge Extract. 2, 672-704. doi: 10.3390/make2040036

NASA GeneLab (n. d.) NASA GeneLab: Open Science for Life in Space. Available online at: https://genelab.nasa.gov/

Nelson, C., Acuna, A., Paul, A., Scott, R., Butte, A., Cekanaviciute, E., et al. (2021). Knowledge network embedding of transcriptomic data from spaceflown mice uncovers signs and symptoms associated with terrestrial diseases. Life 11, $1-14$.

Neutelings, T., Nusgens, B., Liu, Y., Tavella, S., Ruggiu, A., Cancedda, R., et al. (2015). Skin physiology in microgravity: a 3-month stay aboard ISS induces dermal atrophy and affects cutaneous muscle and hair follicles cycling in mice. NPJ Microgravity 1:15002

Opsahl, T., Agneessens, F., and Skvoretz, J. (2010). Node centrality in weighted networks: generalizing degree and shortest paths. Soc. Networks 32, 245-251. doi: 10.1016/j.socnet.2010.03.006

Pellet, J., and Elisseeff, A. (2008). Using markov blankets for causal structure learning. J. Machine Learn. Res. 9, 1295-1342. 
Ram, R., and Chetty, M. (2011). A markov-blanket-based model for gene regulatory network inference. IEEE/ACM Trans. Comp. Biol. Bioinform. 8, 353-367.

Rausch, V., Sala, V., Penna, F., Porporato, P., and Ghigo, A. (2021). Understanding the common mechanisms of heart and skeletal muscle wasting in cancer cachexia. Oncogenesis 10, 1-13.

Réda, C., Kaufmann, E., and Delahaye-Duriez, A. (2020). Machine learning applications in drug development. Comp. Structural Biotechnol. J. 18, 241-252.

Rullman, E., Mekjavic, I., Fischer, H., and Eiken, O. (2016). PlanHab (Planetary Habitat Simulation): the combined and separate effects of 21 days bed rest and hypoxic confinement on human skeletal muscle miRNA expression. Physiol. Rep. 4:e12753. doi: 10.14814/phy2.12753

Smith, R., Cramer, M., Mitchell, P., Lucchesi, J., Ortega, A., Livingston, E., et al. (2020). Inhibition of myostatin prevents microgravity-induced loss of skeletal muscle mass and strength. PLoS One 15:e0230818. doi: 10.1371/journal.pone. 0230818

Syed Sazzad, A., Swarup, R., and Jugal, K. (2020). Assessing the effectiveness of causality inference methods for gene regulatory networks. IEEE/ACM Trans. Comp. Biol. Bioinform. 17, 56-70. doi: 10.1109/tcbb.2018.2853728

Tsamardinos, I., Aliferis, C., Statnikov, A., and Statnikov, E. (2003). "Algorithms for large scale markov blanket discovery," in Proceedings of the Sixteenth International Florida Artificial Intelligence Research Society Conference. (Florida, FL: St. Augustine), 376-381.

Versari, S., Longinotti, G., Barenghi, L., Maier, J., and Bradamante, S. (2013). The challenging environment on board the International Space Station affects endothelial cell function by triggering oxidative stress through thioredoxin interacting protein overexpression: the ESA-SPHINX experiment. FASEB J. 27, 4466-4475. doi: 10.1096/fj.13-229195

Wang, Y., You, Z., Yang, S., Yi, H., Chen, Z., et al. (2020). A deep learningbased method for drug-target interaction prediction based on long short-term memory neural network. BMC Med. Inform. Decis. Mak. 20(Suppl 2): 49. doi: 10.1186/s12911-020-1052-0

Wu, W., Lin, J., Pan, C., Chan, T., Liu, C., Wu, W., et al. (2020). Amplificationdriven BCL6-suppressed cytostasis is mediated by transrepression of FOXO3 and post-translational modifications of FOXO3 in urinary bladder urothelial carcinoma. Theranostics 10, 707-724. doi: 10.7150/thno.39018

Wu, M., Yong, H., Liang, Z., and Yue, H. (2018). "Link prediction based on random forest in signed social networks."in Proceedings - 2018 10th International
Conference on Intelligent Human-Machine Systems and Cybernetics. (China: IHMSC), 251-256.

Wyart, E., Bindels, L., Mina, E., Menga, A., Stanga, S., et al. (2020). Cachexia, a systemic disease beyond muscle atrophy. Int. J. Mol. Sci. 21, 1-18.

Xuan, P., Zhao, L., Zhang, T., Ye, Y., and Zhang, Y. (2019). Inferring drug-related diseases based on convolutional neural network and gated recurrent unit. Molecules 24:2712. doi: 10.3390/molecules2415 2712

Yang, J., Cao, Y R., Li, Q., and Zhu, F. (2018). “Muscle atrophy in Cancer.” In Muscle Atrophy, ed. X. Junjie (New York, NY: Springer), 329-346. doi: 10.1007/ 978-981-13-1435-3.

Yang, Y., Lichtenwalter, R., and Chawla, N. (2015). Evaluating link prediction methods. Knowledge Information Systems 45: 751-782. doi: 10.1007/s10115014-0789-0

Zea, L. (2015). Drug discovery and development in space. Proc. Int. Astronaut. Cong. 1, 273-282.

Zhao, K., and So, H. (2018). Drug repositioning for schizophrenia and depression/anxiety disorders: a machine learning approach leveraging expression data. IEEE J. Biomed. Health Inform 23, 1304-1315. doi: 10.1109/jbhi.2018.2856535

Conflict of Interest: The authors declare that the research was conducted in the absence of any commercial or financial relationships that could be construed as a potential conflict of interest.

Publisher's Note: All claims expressed in this article are solely those of the authors and do not necessarily represent those of their affiliated organizations, or those of the publisher, the editors and the reviewers. Any product that may be evaluated in this article, or claim that may be made by its manufacturer, is not guaranteed or endorsed by the publisher.

Copyright (C) 2021 Manian, Orozco-Sandoval and Diaz-Martinez. This is an openaccess article distributed under the terms of the Creative Commons Attribution License (CC BY). The use, distribution or reproduction in other forums is permitted, provided the original author(s) and the copyright owner(s) are credited and that the original publication in this journal is cited, in accordance with accepted academic practice. No use, distribution or reproduction is permitted which does not comply with these terms. 